\title{
PRESÈNCIA MUSICAL A LA CATEDRAL DE TARRAGONA DURANT LA SEGONA MEITAT DEL SEGLE XVIII A TRAVÉS DE LES SEVES ACTES CAPITULARS
}

\author{
MUSICAL PRESENCE IN TARRAGONA CATHEDRAL \\ IN THE SECOND HALF OF THE EIGHTEENTH CENTURY, \\ ACCORDING TO THE RESOLUTIONS OF ITS ECCLESIASTICAL CHAPTER
}

\author{
Montserrat Canela i Grau \\ Universitat Autònoma de Barcelona
}

\begin{abstract}
Resum:
L'objectiu del present article és col-laborar en la recuperació històrica de la música produïda a la catedral de Tarragona durant la segona meitat del segle XVIII. En aquest cas, per mitjà del buidatge sistemàtic de la informació al·lusiva a la música present en el Llibre esborrany corresponent al període 1764-1779. Aquestes dades permetran obtenir una primera visió de les activitats de la capella de música de la catedral metropolitana de Tarragona i primada de les Espanyes a la segona meitat del segle XVIII. Es tracta d'una de les capelles menys conegudes de Catalunya, tot i que ha estat objecte d'alguns estudis concrets ${ }^{1}$, així com d'algunes tesis doctorals ${ }^{2}$ centrades en els mestres de capella del segle XVIII. Amb la primera publicació del buidatge musical de les actes capitulars s'espera contribuir a la difusió dels aspectes documentals que envolten l'esmentada capella, una de les més destacades de la Catalunya del segle XVIII.
\end{abstract}

1 Vegeu: "La capella musical de la seu de Tarragona a mitjan segle XVIII", Boletín Arqueológico de la Societat Arqueològica Tarraconense, BONASTRE I BERTRAN, Francesc, IV/133-140 (Tarragona, 1976-1977): 259-270. "Canonges, comensalies, beneficis, mestres de capella i organistes de la catedral de Tarragona", Butlletí Arqueològic de la Reial Societat Arqueològica Tarraconense, RAMON I VINYES, Salvador, V/21-22 (Tarragona, 1999): 241-595. "Joan Crisòstom Ripollès (†1746): Biografia i catalogació de la producció musical conservada”, Boletín Arqueológico de la Societat Arqueològica Tarraconense, GREGORI CIFRÉ, Josep M., IV (Tarragona, 1976-1977): 251-258. "Joan Crisòstom Ripollès (ca.1680-1746), Mestre de capella de la catedral de Tarragona", Recerca musicològica, GREGORI CIFRÉ, Josep M., II (Barcelona, 1982): 19-41. "Joan Crisòstom Ripollès (1678-1746) a les oposicions del magisteri de capella de la seu de Tortosa”, Butlletí Arqueològic de la Reial Societat Arqueològica Tarraconense, GREGORI CIFRÉ, Josep M., V/14 (Tarragona, 1992): 137-147. "Mira Piadosa Thecla, de Melcior Juncà (1757-1809): contribució a l'estudi del villancet català de les darreries del segle XVIII", Recerca musicològica, RUSIÑOL I PAUTES, M. Carme, VIII (Barcelona, 1988): $143-191$.

2 Vegeu: RIFÉ I SANTALÓ, Jordi, Els villancets d'Enmanuel Gònima, 1712-1792: un model de transició musical del barroc al preclassicisme a la Catalunya del segle XVIII. Tesi doctoral, Universitat Autònoma de Barcelona, 1992. RUSIÑOL I PAUTES, M. del Carme, Els villancets de Melcior Juncà: contribució al seu estudi a la música catalana del segle XVIII. Tesi doctoral, Universitat Autònoma de Barcelona, 1994. CAZURRA BASTÉ, Anna, El compositor i mestre de capella Joan Rosell i la seva aportació al preclassicisme musical hispànic. Tesi doctoral, Universitat Autònoma de Barcelona, 1994. FA ECHEVARRIA, Mayra, Melcior Juncà (1757-1824): biografia i música litúrgica. Tesi doctoral, Universitat Autònoma de Barcelona, 1999. 


\title{
Paraules clau:
}

Actes capitulars, catedral de Tarragona, capella de música, segle XVIII

\begin{abstract}
The aim of this essay is to contribute to the historical recovery of the music composed in Tarragona Cathedral in the second half of the $18^{\text {th }}$ century. This recovery was made possible through the systematic extraction of information regarding the music existing in the Rough Book belonging to the period between 1764 and 1779. This information will enable us to have a preliminary idea of the activities which took place in the music chapel of the Metropolitan Cathedral of Tarragona and Primacy of Spain during the second half of the $18^{\text {th }}$ century. This is one of the lesser known chapels in Catalonia. However, it has been the object of some specific studies as well as some $\mathrm{PhD}$ theses focusing on the figures of $18^{\text {th }}$ century chapel masters. The first publication of the extracted music from the Chapter House Acts will contribute to the expansion of knowledge about the documents from the aforementioned chapel.
\end{abstract}

Key words:

Chapter House Acts, Tarragona cathedral, music chapel, $18^{\text {th }}$ century

\section{Resumen:}

El objetivo del presente artículo es colaborar a la recuperación histórica de la música producida en la catedral de Tarragona en la segunda mitad del siglo XVIII. En este caso, a través del vaciado sistemático de la información alusiva a la música contenida en el Libro borrador correspondiente al período 1764-1779. Esta documentación permitirá obtener una primera visión de las actividades de la capilla de música de la catedral Metropolitana de Tarragona y Primada de las Españas durante la segunda mitad del siglo XVIII. Se trata de una de las capillas menos conocidas de Cataluña, a pesar de que ha sido objeto de algunos estudios concretos así como de algunas tesis doctorales centradas en las figuras de los maestros de capilla del siglo XVIII. Con la primera publicación del vaciado musical de las actas capitulares se espera contribuir a la difusión de los aspectos documentales alrededor de la citada capilla, una de las más destacadas de la Cataluña del siglo XVIII.

Palabras clave:

Actas capitulares, catedral de Tarragona, capilla de música, siglo XVIII

\section{INTRODUCCIÓ}

La capella de música de la catedral de Tarragona és una de les grans desconegudes de Catalunya malgrat els estudis que se li han dedicat fins al moment. La idea parcial i fragmentada que se'n té ve donada per la poca documentació impresa de què es disposa i pel fet que la catedral, en el decurs de la seva existència, ha sofert diversos incendis i espolis que han portat a la pèrdua de valuosos manuscrits que, sens dubte, ajudarien a tenir una idea més precisa de les funcions específiques de la capella.

Amb el present buidatge documental del Llibre esborrany corresponent al període 1764-1779³ s'aporta una primera informació sobre músics cantors i instrumentistes, activitats de la capella i altres dades. Per la seva natura d'esborrany, el volum del qual s'ha extret l'esmentada informació presenta dades recollides rapidament amb la idea de ser passades a net posteriorment al Llibre d'Actes Capitulars.

3 Els materials que aquí presento són fruít de dos anys de treball a l'Arxiu Històric Arxidiocesà de Tarragona (AHAT) i formen part de la meva tesi doctoral, en curs. Agraeixo al Dr. Antonio Ezquerro Esteban els consells per a la el-laboració del present article. Així mateix, agraeixo al director de l'Arxiu Històric Arxidiocesà de Tarragona i canonge arxiver de la catedral, Mn Manel Fuentes totes les facilitats de consulta i recolzament. 
És per això que la grafia és poc legible en molts casos. El volum no està ordenat correctament per ordre cronològic, de manera que és possible trobar en un mateix full anotacions d'anys diferents. Això pot ser degut precisament al fet de tractarse d'un esborrany (i per tant, al fet que els materials ja serien ordenats posteriorment) així com al fet de no voler malgastar paper.

Tot i això, per a la seva publicació s‘han ordenat els materials que es presenten seguint una numeració anual:

- Any 1764: pàgs. 438-613, pp. 611-616, i pp. 631-650

- Any 1765: pàgs. 334-429, i pp. 617-630

- Any 1766: pàgs. 187-316, i pp. 429-437

- Any 1767: pàgs. 0-182, i pp. 316-332

- Any 1768: pàgs. 182-186

- Any 1777: pàgs. 651-789

- Any 1778: pàgs. 789-914

- Any 1779: pàgs. 655-658, pp. 914-917

A continuació es transcriuen les corresponents resolucions capitulars, escrites bàsicament en català. Com a criteris de transcripció s'han emprat els habituals per a aquest tipus de documentació ${ }^{4}$. Al final s'hi ha afegit un índex onomàstic amb els noms pròpis que es citen en les actes i un índex de matèries (amb els càrrecs musicals, instruments i matèries) per tal de facilitar la consulta.

\section{LLIBRES D'ACTES CAPITULARS}

[Esborrany dels anys 1764, 1765, 1766, 1767, 1777, 1778, 13-1-1779] ${ }^{5}$

[ 1 ] Pàg. 582 : «Die 1 8us 1764»: "Dictus Dnus Arch" mor $\operatorname{Sin}^{\text {b }}$ prop $^{\text {b }}$ Que de alguns / añs a esta part ha advetit que lo Anton Gassol / sustentor primer de V.S fa moltas ausencias / del Chor per a con-

4 Tot el que s'escriu entre claudàtors és del transcriptor. Els "marginalia" (anotacions manuscrites originals anotades al marge) s'indiquen en cada acta capitular a prop del lloc on apareixen (a vegades al començament de l'acta, d'altres al llarg del cos de text), entre cometes. Es diferencien les línies del manuscrit pel procediment habitual de les barres inclinades. Els fragments que aporten poca o cap informació musical s'han marcat al text a través de punts suspensius entre claudàtors. Es dóna la paginació original a l'inici de cada entrada. Cada acta capitular s'ha numerat adjudicant-li aquí un número d'entrada entre claudàtors i en negreta, per tal de facilitar la cerca dels noms propis. Les ratllades, els subratllats i les abreviatures responen al que s'ha trobat en la corresponent acta capitular. El cos de text de cada acta s'escriu entre cometes.

5 Llibre manuscrit de 325 × $225 \mathrm{~mm}$, enquadernat cosit, amb tapes de cartró folrades de pergamí de color clar; en el llom s'hi pot llegir: «Borrador / Resolucion: / capitul [atio] / 1764, 65, / 66, 67, 77 y 78, / ? / A / 53»; a la coberta hi ha una etiqueta manuscrita en la qual es pot llegir: «lletra $\mathrm{A} \mathrm{N}^{\mathbf{0}}$ [93]3 / Libro brorrador de acuer- / dos capitulares tomados en / los años 1764.65.-66.- / y 67. y 1777 y 78 hasta el / dia 13 de Enero de 1779. Des / de el 1764 hasta el 1767 no /estan en orden cronologico». El Llibre esborrany es tanca amb dos cordons. Consta de 917 folis de paper, anotats amb tinta negra de diverses mans, dels quals n'hi ha 6 en blanc que estan numerats: pàg. 175, 193, 199, 205, 373 i 459. Per la seva natura d'esborrany, el llibre presenta diversos folis blancs sense numerar: tretze al començament i un al final. L'enquadernació, feta amb quadernets, deixa també un nombre variable de fulls en blanc al començament o final d'aquests. 
correr a [?] festas majors / de varios Pobles del $\operatorname{Arch}^{t}$ y que compren / que se li concedeix aquest permis per a / sucorrer sa necessitat ab lo que ditas fun- / cions li produeixen. Y com la falta de / dit sustentor es notabilissima en lo Chor / apar se li podria donar de limosna fins / a mas $10 £$ annuals de la $\mathrm{Adm}^{\circ} \mathrm{de}$ la Obra y privarlo de / estas ausencias, et dnus $\mathrm{Cap}^{\mathrm{s}} \operatorname{Det}^{\mathrm{t}}$ que se / fasse con se ha proposat sen que ser- / vesca de exemplar. Et in continenti Dnus Can ${ }^{\mathrm{s}}$ Baldrich dixit / que apar [?] la mateixa raho / [Pàg. 583 :] ab las frequents ausencias dels Monacillos de V.S / per a acudir a semblants funcions, lo que ames de / ser notable per la falta que fan a V.S es de / algun deshonor a la Igla el ferse publich / que se permet esta concurrencia a aquellas / festas per ajuda de costa al Mestre de Capella / de [?] Y se evitaria una cosa y altre si al / dit $\mathrm{M}^{\mathrm{e}}$ de Capella manava V.S subminis- / trarli annualm ${ }^{\mathrm{t}}$ unas $25 £$ que es lo que / poden guañar los referits monacillos en / la sobredita concurrencia de festas. Et Dnus / Cap ${ }^{\mathrm{s}}$ Det $^{\mathrm{t}}$ que se fasse com se ha proposat / donantse $25 £$ de la Obra annualmen ${ }^{t}$ a / dit $\mathrm{M}^{\mathrm{e}}$ de Capella. Dictus Dnus Can ${ }^{\mathrm{s}}$ Baldrich prop ${ }^{\mathrm{t}}$ Que Anton / Montaña primer segon violi de la capella / de V.S li ha dit que ab lo curt salari de $£ 30$ / li es impossible mantenirse en lo servey / de V.S Y que li proposa una conveniencia molt major en Madrit pero que desitjant / servir a V.S se quedaria ab gust com V.S / li aumentas lo salari de manera que / tal qual pugues mantenirse en esta Ciutat / [Pàg. 584 :] Y que havent parlat ab lo Mestre de Capella sobre / aquest particular li ha assegurat que la / Capella quedara molt mal servida sens la con- / currencia de un segon primer segon violi ja sia / de Montaña o altre pero que qualsevol / foraster ab igual habilitat a la de Montaña / no lo pod. Et ra V.S mantenir en esta Ciutat ab / lo que se que se quedara dit Montaña Et Dnus / $\mathrm{Cap}^{\mathrm{s}} \operatorname{Det}^{\mathrm{t}}$ que se li donen annualm ${ }^{\mathrm{t}}$ fins / a $70 £$ annualm $^{\mathrm{t}}$ de la $\mathrm{Adm}^{\circ}$ de la obra. Per ocasio de tractarse de cosas de capella lo $\mathrm{S}^{\text {or }}$ / Cang ${ }^{\mathrm{e}}$ Foguet feu pnt que lo organista / te obligacio de enseñar de orga als que se / vullan apendrer de esta Ciutat majorm / als que han servit a V.S de monacillos ; Y / que aquest exercici lo practica dit Orga- / nista ab molta [?] y apar seria / just que V.S li manas recordar esta sa / $\mathrm{oblig}^{\mathrm{o}}$. Et Dnus $\mathrm{Cap}^{\mathrm{s}} \operatorname{Det}^{\mathrm{t}}$ que los $\mathrm{S}^{\text {ors }} /$ Sindichs se servescan recordar esta oblig / a dit Organista."

\section{Any 1765}

[ 2] Pàg. 626 : «D. 15 Janer 1765»: "Casanova que un escolanet dit Jph solanellas / va perdent la veu y que te Jph Oliver na- / tural de Reus."

[3] Pàg. 336 : «Die 4 febrer 1765» : "Casanova Que los Adm ${ }^{\text {ors }}$ no pugan cobrar la / ultima tercia dels arrendaments que los arren- / dataris aporten recibo del $\mathrm{Adm}^{\text {or }}$ de la cera y / del $\mathrm{M}^{\mathrm{e}}$ de Capella Y que lo $\mathrm{Adm}^{\circ}$ de la obra [?] / la cera y obra que hage cobrat / Que los Adm ${ }^{\text {ors }}$ degan donar comptes dins dos / mesos de finida la $\mathrm{Adm}^{\circ}$."

[ 4] Pàg. 339 : «Die 23 february 1765»: "Botines que lo Semmaner Arnet esta impossibilitat per / la poca vista que te de continuar dit offici: Y demana / una subvencio y continuar a tocar lo violo. Dni que / se admet la renuncia Y que los $S^{\text {ors }}$ Sindichs / busquen subjecte Y que se done de la obra $15 £$ / yque inverinam lo aparega als $\mathrm{S}^{\text {ors }}$ Sindichs / y Adm ${ }^{\text {or }}$ y que en lo interim lo altri [ Marge : "Semmaner / asistia a la / Missa matinal / suplesca per / dit Arnet" ]." 
[5] Pàg. 352 : «Die 15 Aprily 1765» : "Dictus Dnus Cans Botines Sind" propt que V.S se / ha de servir resoldrer si se donara la Distrib / [Pàg. 353 :] al que toca lo violo M $^{\mathrm{n}}$ Arnet [ Marge : "en las horas / que residesca"] aixi com las / [?] a mes com a Semmaner Et Dni Cap / Det ${ }^{1}$ que se li done."

[ 6] Pàg. 355 : «Die 19 Aprily 1765»: "Dnus Can ${ }^{\mathrm{s}}$ Botines Sind prop $^{\mathrm{t}}$ Que Fran ${ }^{\mathrm{co}}$ Hernandez / altre dels Mussichs de la Capella de V.S. li ha entregat / una sup ${ }^{a} p^{r}$ V.S. $q^{e}$ si V.S. gusta se llegira [ Marge : "[?]/ Fran ${ }^{\mathrm{co}}$ Ernan- / dez n ${ }^{\mathrm{o}} 26^{\prime}$ ] $\gamma$ Qua lecta Dni Cap determ $^{\mathrm{t}} \mathrm{q}^{\mathrm{e}}$ se li done tota la / Carn que necessite pera y alguna cosa mes per / algun refresch."

[ 7] Pàg. 364 : «D. 9 7 bris 1765» : "Dnus Can ${ }^{s}$ Llorens Sind prop $^{t}$ Que haventse / practicat alguna dili$\mathrm{g}^{\mathrm{a}} \mathrm{p}^{\mathrm{r}}$ lo $\mathrm{S}^{\text {or }}$ Botines y sa / Merce pera fer entrar en lo Hospital de / Misericordia de Barna la filla del Sustentor / Anton Gassol se hi ha trobat algun Inconve- / nient y los apar podentse posar en lo Hospital / de Horfens de la pnt Ciu ${ }^{t}$ y lo un dels Minors / al Hosp ${ }^{1}$ dels Horfens y lo altre en casa lo / $\mathrm{M}^{\mathrm{e}}$ de Capella. Per assó es menester vestirlos / y apar $\mathrm{q}^{\mathrm{e}}$ al $\mathrm{M}^{\mathrm{e}}$ de Capella se li / podria donar alguna cosa de ajuda de costa / $\mathrm{p}^{\mathrm{r}}$ los manteniments del xich per no ser encara / de servey pera la Capella y tambe se haura de / gastar alguna cosa $\mathrm{p}^{\mathrm{r}}$ vestirlos. Et Dni Cap $\operatorname{det}^{\mathrm{t}} / \mathrm{q}^{\mathrm{e}}$ los $\mathrm{S}^{\text {ors }} \mathrm{Sind}^{\mathrm{s}}$ executen y gasten lo $\mathrm{q}^{\mathrm{e}}$ los / aparega necessari de la $\mathrm{Adm}^{\mathrm{o}} \mathrm{de}$ la Carniceria.

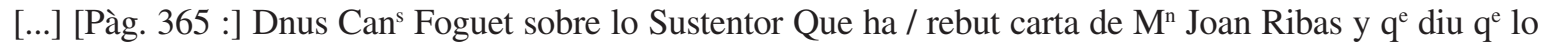
sustentor / ja esta probat y es habil tant de cant pla com de / figurat, y q aixi V.S. determ[in]e lo q e se haura

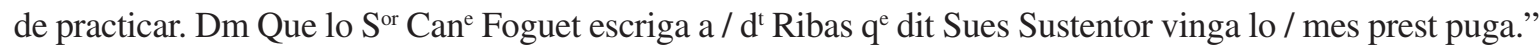

[ 8] Pàg. 367 : «D. 17 7 $7^{\text {re }}$ 1765» : "Dnus Cans Casanovas Adr opere / prop ${ }^{\mathrm{t}}$ Que se li ha proporcionat ocasio de un / niño $\mathrm{p}^{\mathrm{r}}$ monecillo; Y com ni hage dos que / dins poch temps deuran despedirse $\mathrm{p}^{\mathrm{r}}$ haver / perdut la veu si apar a V.S. entrara / $\mathrm{p}^{\mathrm{r}} \mathrm{q}^{\mathrm{e}}$ V.S. lo puga ohir cantar y en cas de gus- / tarli la veu resoldrer $\mathrm{q}^{\mathrm{e}}$ se admettia d $\mathrm{d}^{\mathrm{t}}$ Mone- / cillo. Et Dni determ ${ }^{\mathrm{t}} \mathrm{q}^{\mathrm{e}}$ entre y havent entrat / en lo Chor Jpht Malé natural de Cervera y Cantat ha resolt sa Sen $^{\mathrm{a}} \mathrm{q}^{\mathrm{e}}$ se admetia d Monecillo."

[ 9] Pàg. 369 : «D. 21 7 $7^{\text {re }}$ 1765»: "Dnus Cans Botines prop ${ }^{\text {t }}$ Que lo actual Succentor / preten la porcio de la porsio del Canonicat Penitencier / [Pàg. 370 :] q es pertañia al altre Succentor sino vacava la Suc- / centoria y apar fora mes propi $\mathrm{q}^{\mathrm{e}}$ esta porcio se / repartis entre $\mathrm{M}^{\mathrm{n}}$ Bernat Crespo y lo Semmaner / $\mathrm{M}^{\mathrm{n}}$ Martorell. Et Dni determ ${ }^{\mathrm{t}}$ Que aquella porcio se done a $\mathrm{M}^{\mathrm{n}}$ Bernat $\mathrm{p}^{\mathrm{r}}$ ser lo $\mathrm{q}^{\mathrm{e}}$ continuam ${ }^{\mathrm{t}}$ resideix / y cumpleix la falta de Succentor y no ferho aixi $\mathrm{M}^{\mathrm{n}}$ Martorell Semmaner."

[ 10 ] Pàg. 394 : «Cap del dia 25 8 bris 1765»: "Dnus Dnus Can Botines Sind" prop ${ }^{\mathrm{t}}$ Que Mn / Obia li ha dit que algunas faltas se / feyan al campanar las causavan los que / tenen cordas y particularm ${ }^{\mathrm{t}}$ Onofre / Corbella y lo sastre Donato, Y aixi V.S. se / ha de servir pendrar alguna providencia. / Et Dnus Cap ${ }^{1}$ Det $^{t}$ Que los $S^{\text {ors }}$ Sindichs / se Servescan amonestar a dits campaners / que el que fase falta / se li llevara la corda y que venint es- / cas se servescan los $\mathrm{S}^{\text {ors }}$ Sindichs buscar / Subgecte per dit empleo. [...] [Pàg. 396 :] Dnus Can ${ }^{\mathrm{s}}$ Casanova Admor ${ }^{\mathrm{r}}$ Opere et fabrica / prop $^{\mathrm{t}}$ Que per ocasio de estar malalt un / Monacillo, passa a visitarlo y repara la / incomoditat gran en que estan per dormir / Y si se feia un quarto en un corralet contiguo / a la casa del Mestre se remediaria q lo / que no sera cosa de molt cost Y si es del / agrado de V.S. se executara. Et dnus Cap ${ }^{1}$ Det $^{\mathrm{t}}$ Placet."

[ 11 ] Pàg. 397 : «D. $308^{\text {bris }} 1765 »$ : "Dnus Cans Botines Sindicus prop ${ }^{\text {t }}$ Que lo / Mestre de Capella li ha entregat un pa- / per de Informe de dos subgectes de / Bara que li havian suposat eren bons / per 
Sustentors de esta Igla y que saben / de cant figurat y pla que si V.S. gusta / se llegira Dnus leg ${ }^{\mathrm{r}}$ Quo lecto Dnus Cap ${ }^{1} /$ Det $^{t}$ Que los $S^{\text {ors }}$ arxivers se serves- / can escriurer a $\mathrm{D}^{\mathrm{n}}$ Juan de Ribas / que se assegure si lo un de dits dos Sub- / gectes anomenat Mathias N. actual / Musich de la Igla del Pi desitja ser / Sustentor de esta igla y en cas de / volerho lo fasse examinar de la / veu tant de cant figurat com de / cant pla y sapia si la Veu, encara que / se diu ser veu de coll / [Pàg. 398 :] es bastant per Sustentor y si esta ho es y / lo proban habil tant de cant figurat com del / pla lo fasse venir ab la major brevetat."

[ 12 ] Pàg. 402 : «D. 22 9 $9^{\text {bris }}$ 1765»: "Dnus Can ${ }^{\mathrm{s}}$ Llorens Sinds prop ${ }^{\mathrm{t}}$ Que se han / rebut dos cartas per V.S. de $\mathrm{D}^{\mathrm{n}}$ Joan de Ribas / Sindich de V.S. en Bar ${ }^{\text {na }}$ de data la una de 16 y altra de 19 dels corrents que / [Pàg. 403 :] si V.S. gusta se llegiran ${ }^{60}$ Dni $\gamma$ Determ ${ }^{t}$ que / los Sors Arxivers se servescan respondrer / a dit $\mathrm{S}^{\text {or }} \mathrm{D}^{\mathrm{n}}$ Joan que considerant sabria / tant fundat lo dictamen del $\mathrm{S}^{\mathrm{r}}$ Prat en / punt de la causa de Ferrando se con- / forma gustos sa Sria a ell. En quant a Sustentor que se li responga / que se te noticia de altre Subgecte y que sa Sria se esta informant de sas cir- / cunstacias per si podra servir a / esta Igla; no obstant podra dit $\mathrm{S}^{\text {or }} / \mathrm{D}^{\mathrm{n}}$ Joan informarse dels concurrents / en la oposicio de la Capellania vacant / en la Igla del Pi y se servesca avisar / si algun de ells seria habil per a re- / gentar la Sustentoria vacant."

[ 13 ] Pàg. 647 : «D. 29 X bris a Natte 1765»: “Arch" Mar Que se han rebut dos Cartas / una de Anton Llobet y altra de Sagimon / Llobet dadas en Barna ã 22 del corrent / Dnus Que se retornen las bonas Pasquas / $\mathrm{Arch}^{\mathrm{s}} \mathrm{Ma}^{\mathrm{r}}$ Que Pere Joan Aubia Diaca / y Benat de esta Igla y Segon Campaner / de ella li ha entregat una Sup ${ }^{a} p^{r}$ V.S. en / la q $q^{e}$ suplica a V.S. se digne elegirlo en campa- / ner major $\mathrm{q}^{\mathrm{e}}$ vaca $\mathrm{p}^{\mathrm{r}}$ dismissio $\mathrm{q}^{\mathrm{e}}$ de dit ofici ha / fet $\mathrm{M}^{\mathrm{n}}$ Matheu Claramunt. $\mathrm{D}^{\mathrm{s}} \mathrm{D}^{\text {nus }} \mathrm{Arch}^{\mathrm{s}} \mathrm{Ma}^{\mathrm{r}}$ prop ${ }^{\mathrm{t}}$ Que Thomas Marti / Clergue natural de esta $\mathrm{Ciu}^{\mathrm{t}}$ y Bernat de Yvissa / li ha entregat altre Mem ${ }^{1}$ en lo qual / sup ${ }^{c a}$ a V.S. anomenarlo Campaner major o / altre offici q $q^{\mathrm{e}}$ sia del agrado de V.S. Quals / suplicas si V.S. gusta se llegira. Dni Leg / Determ ${ }^{\mathrm{t}}$ y elegiren en Campaner major de la / pnt $S^{\text {ta }}$ Igla a dit $\mathrm{R}^{\mathrm{m}}$ Pere Joan Aubia Diaca / Benat y en monjo Porter de Chor y segon / Campaner a dit Thomas Marti Clergue durante / beneplacit donant y concedint a cada un dels / referits tots los salaris honors y exempcions que / a semblants officials se han acostumat concedir."

\section{Any 1766}

[ 14 ] Pàg. 437 : «Die 18 Janer 1766» : "Llorens Que no obstant que V.S. aproba al Sustentor y que / dona esperansas de ser així per nadal, haventse fet / aguna diliga ab lo coronel y apar [ Marge : "Que no si pensa / ab dit Sustentor / y que se posan / edictes" ]."

[ 15 ] Pàg. 189 : «Die 1 Fabruary 1766» : "D Cans Llorens Sins proposuit qe lo Mestre de / Capella dificulta si demà $q^{e}$ es la Verge y no pot entrar / per ser Dominica privilegiada deura cantar la Capella / D.D. $\mathrm{q}^{\mathrm{e}}$ no. Dictus Llorens $\operatorname{Sin}^{\mathrm{s}} \mathrm{q}^{\mathrm{e}}$ respecte las vacans de / Sustentor y Semaner si pereixia a V.S. podria substituhir/ [Pàg. 190 :] per este $\mathrm{M}^{\mathrm{n}}$ Ordeix y per aquell M ${ }^{\mathrm{n}}$ Armet."

[ 16 ] Pàg. 190 : «Die 3 Fabruary 1766»: "D Llorens $\operatorname{Sin}^{\text {s }}$ Que lo $\mathrm{S}^{\text {or }} \mathrm{D}^{\mathrm{n}}$ jaume Fer- / rer á rebut una carta del coronel de Fusillers / en q diu se interessia ab V.S. peraq ${ }^{\mathrm{e}}$ suspenga / anumenar Sustentor respecte $\mathrm{q}^{\mathrm{e}}$ dins breus dias / donara las llisencias á Silverio Vila sargento de / son Regim ${ }^{\mathrm{t}} \mathrm{y}$ ja las tindria 
anover estat encar- / regat de la Vantera de las Recultas [sic."Bandera de las reclutas"] en atencio / qe es subjecta $q^{\mathrm{e}}$ preten dit empleo de Sustentor / y abrobat ja per V.S. en cuya consideracio si / apar bé a V.S. podria suspendresse lo despatg / de edictes progectat. $\mathrm{D}^{\mathrm{ni}} \mathrm{q}^{\mathrm{e}}$ se suspengan los edictes y se espera a / Silverio Vila Servinsa lo $S^{\text {or }} \mathrm{D}^{\mathrm{n}}$ Jaume es- / criuen las gracias al Coronel suplican la breve- /dad."

[ 17 ] Pàg. 203 : «Die 15 Abril 1766» : "Botines sobre la xica del Sustentor que se li / continue en [pagar] la [despesa] a las Horfenas."

[ 18 ] Pàg. 212 : «Die 10 Maig 1766» : "Dnus Cans Casanova Admº opere prop" / Que Fran ${ }^{\text {co }}$ barcelo y Miguel Jovena / li han entregat quiscun una suplica / per V.S. que si V.S. gusta se llegira / [Pàg. 213 :] Dni legant ${ }^{\mathrm{s}}$ quibus lectis Dni Cap ${ }^{1}$ elegiren / y anomenaren a dit Fran ${ }^{\mathrm{co}}$ Barcelo en Subs- / titut de Jph Guasch Musich de trompa / y clarin sens percepcio de salari / quedant este a favor de [?] Guasch, y conce/ direm a dit Barcelo la futura succesio / ab lo mateix salari, franquesas y exencions / que logra dit Guasch, durante beneplacito. Y que lo $\mathrm{S}^{\mathrm{r}}$ Obrer diga a $\mathrm{M}^{\mathrm{n}}$ Joan Ordeig / que cumple en son offici de tocar lo fogot."

[ 19 ] Pàg. 223 : «Die 7 Juny 1766»: "Botines que lo Sustentor mes antich demana una / gratificacio Dni comº als Sindichs y Obrers / per a gratificarlo. [Pàg. 224 :] Dictus Dnus Can ${ }^{\mathrm{s}}$ Botines sindicus proposuit / Que lo Sustentor mes antich apar es digne / de alguna gratificacio per lo temps ha / suplert per la falta del altre, y per lo / que actualm ${ }^{t}$ treballa per no ser lo modern / Sustentor bastant habil per a regir / lo Cor, com dit Sustentor ho suplica a V.S. / Et Dni Cap ${ }^{1}$ Det $^{t}$ que se gratifique / a disp ${ }^{\circ}$ dels S $^{\text {ors }}$ Sindichs y Obrers."

[ 20 ] Pàg. 229 : «Die 16 Setembre 1766»: “El S ${ }^{\text {or }} \mathrm{D}^{\mathrm{n}}$ Pedro ignacio Perello propuso: Que alos / 18 del mes de Marzo de este año por muerte de $/ \mathrm{M}^{\mathrm{n}} \operatorname{Fran}^{\text {co }}$ Escola saco un beneficio de esta $\mathrm{S}^{\text {ta }}$ Igla cuya / presentacion especta al canonigo turnario q es su Mer / Que ha suplicado [ Marge : "varias veses"] al $\mathrm{Cav}^{\mathrm{do}}$ se habriesse el truno para / presentar sugeto al Dho beneficio y $\mathrm{q}^{\mathrm{e}}$ por motivos $\mathrm{q}^{\mathrm{e}} /$ ha tenido su Sen ${ }^{\mathrm{a}}$ no lo ha logrado; Y como el tiempo de presentar feneze el dia 18 de este, suplica nuevam ${ }^{\text {te } / \text { a su Sen }}$ / se habra dho turno. Ygualm ${ }^{\text {te }}$ por muerte / de $\mathrm{D}^{\mathrm{n}}$ Nicolas Ametller Arzediano de Villaseca / de esta misma Ygla acahecida el 10 del corriente / mes, vaca dho Arzedianato, $q^{\text {e }}$ segun el estado de / la ygta, apoyado con Bulas App ${ }^{\text {cas }}$ prettende igualm ${ }^{\text {te }} /$ obrar el referido Canonigo Prello como mas antiguo / y para diho efecto suplico tambien se abriese el turno acostumbrado; y ohida dha proposicion por los $\mathrm{S}^{\text {es }}$ Capitulares, attendiendo haver acudido / antes de ahora a los pies del Rey nuestro $S^{\text {or }}\left(q^{\mathrm{e}} /\right.$ Dios $\left.\mathrm{g}^{\mathrm{e}}\right)$ afin de inclinar a la piedad de su Magd a disponer y resolver su nuevo estado en esta / $\mathrm{S}^{\text {ta }}$ Ygla, en el qual como a objeto principal, se / suplica la Union de las rentas de los Dignidades / a la Mensa Cap ${ }^{\mathrm{r}}$ y en seguida la extincion / de algunas piezas de dhas Dignidades y Canongias / y aplicacion a la capilla de Mussicos y a nuevos [Pàg. 270 :] Parrochos de la Ciu[da]d Porcion de Raziones y bene- / ficios y la extincion o supression de muchos de estos: Su Mag ${ }^{d}$ mandó a la Camara q e viesse el / expediente: la Camara lo passó al $\mathrm{S}^{\text {or }}$ Fiscal, este / ha tratado y arreglado segun su parezer el re / ferido estado q $\mathrm{q}^{\mathrm{e}}$ passó despues al Y11 ${ }^{\mathrm{mo}}$-Sor-Arzopo / de esta Igła dio su dictamen que la orden de la misma / [ Marge : "Se comunicó á / todos los Inte- / ressados"] estando las cosas en este estado / vacaron el ella la dignidad de Chantre y una Ca- / nongia por muerte de $\mathrm{D}^{\mathrm{n}} \mathrm{Jph}$ de Toda / otra canongia por renuncia de $\mathrm{D}^{\mathrm{n}}$ Fran ${ }^{\mathrm{co}} /$ Sebrian en Meses aetrdim reservados a su / Mag ${ }^{\mathrm{d}} \mathrm{a}$ quien rendidam ${ }^{\text {te }}$ suplicamos $\mathrm{q}^{\mathrm{e}}$ se / dignasse suspender la provission de dhas piezas / hasta $\mathrm{q}^{\mathrm{e}}$ hubiesse 
resuelto su Magd ${ }^{\mathrm{d}}$ sobre la / nueba planta; y condecendió benignam ${ }^{\text {te }}$ su / Mag ${ }^{\mathrm{d}}$ a nuestros ruegos: En esta Intelligencia / no podemos dar lugar a la abertura de turno / para la presentacion al beneficio ni para la obsion de la Dignidad, hasta representar á / su Magd este suscesso con la ingenuhidad y / verdad propia de nuestro Caracter y nos con- / formaremos, como debemos con la resolucion de su Magd reservando a Dho Canonigo $\mathrm{D}^{\mathrm{n}}$ Ignacio / Perello todo el año por qualquier motivo pueda / pertenecerle, tanto en [ Marge : "el nombram ${ }^{\text {to }}$ / de la"] Persona para el [Pàg. 271 :] beneficio, como en la obsion de la Dignidad, sin q / la Dilacion precissa de esta representacion / le pare perjuizio el mas minimo á la percepcion / de los frutos de la Dignidad, en caso de verificarse / segun las $\mathrm{R}^{\mathrm{s}}$ inttenciones la obsion $\mathrm{q}^{\mathrm{e}}$ prettende / en aquella." [I d'una altra mà posterior: "y que se li done copia"]

\section{Any 1767}

[ 21 ] Pàg. 53 : «Die 9 Mary 1767»: "Deulofeu que lo Mestre de Capella desitjaria lo hortet / del Sustentor per tenir mes ambits y ferli un / corralet Dmi placet." [Pàg. 55 :] "Dictus Dmu ${ }^{\mathrm{s}} \mathrm{Can}^{\mathrm{s}}$ Deulofen $\mathrm{Adm}^{\text {or }}$ opere / prop $^{\mathrm{t}} \mathrm{Que}$ lo Mestre de Capella desitja / tenir uns pochs mes de ambits en la casa del / Magisteri, y tenirhi un corralet el que lograria / si V.S. se dignava resoldrer el que se / añadis a dita casa lo hortet del sustentor segon, pues a este no li es de util algun".

[ 22 ] Pàg. 27 : «Die 14 Mary 1767» : [ Marge : "Collocar en lo Chor al Arpista / y Baxonista" ] "Botines sobre la [?] de posar al Chor lo Arpista y / baxonista no essent Bene[fici]ats : lo Arpista al / 1 Chor, y lo baxonista al 2. [...] [Pàg. 32 :] [ Marge : "Sobre estar en / lo Chor lo arpista / y baxonista que / no eran beneficiats" ] Dictus Dnu ${ }^{\mathrm{s}}$ Can Botines Sind ${ }^{\mathrm{s}}$ Prop $^{\mathrm{t}}$ Que en dias passats / M ${ }^{\mathrm{n}}$ Matheu Claramunt repugna posarse / al faristol, ab lo motiu de que $\mathrm{M}^{\mathrm{n}}$ Arnit / que ocupa lo lloch de Arpista, y lo baxonista no essent Bene[fici]ats devian estar un a cada / Chor y no tots dos en un mateix Chor ab / que VS se ha de servir pendrer resolució so / bre est punt. Dm Cap Dert. Que los sobredits / no essent Bene[fici]ats deuhen estar un en cada / Chor".

[ 23 ] Pàg. 72 : «Die 5 Juni 1767»: "Dms Cans Botines Sind" Prop" Que segons la / serie de las fundacions fetas per los $S^{\text {ors }}$ Marm / del $\mathrm{g}^{\mathrm{o}}$, $\mathrm{S}^{\text {or }}$ Ardiaca Ameller, la primera es la del / Aniversari, y apar podria dotarse ab $30 £$ / Annuals distrib ${ }^{\mathrm{s}}$ en esta forma ço es al cele / brant per la caritat de la Missa : 15 ş

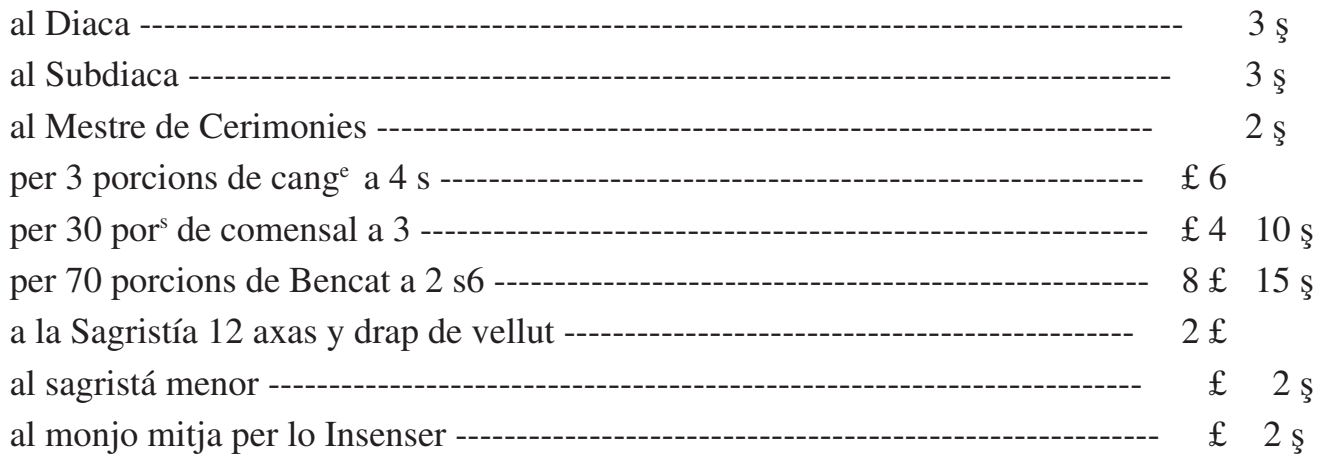




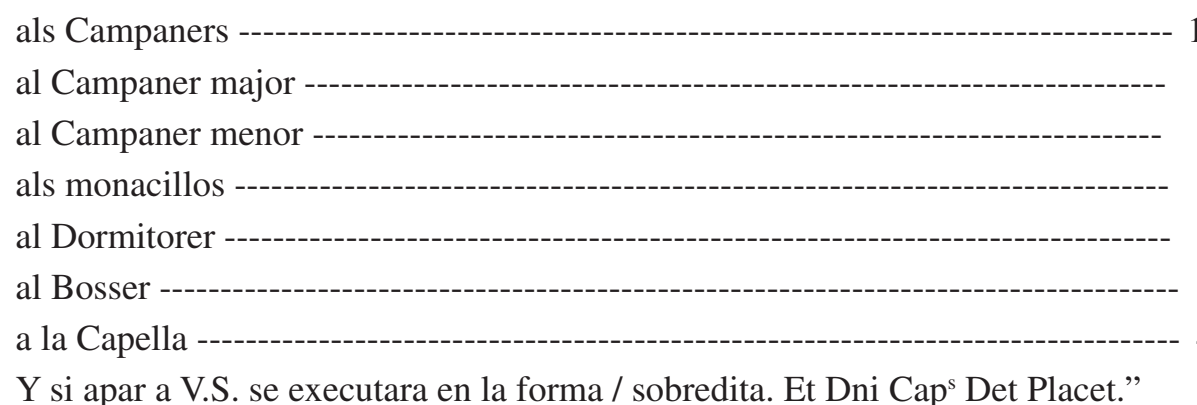

$\begin{array}{rr}1 £ & 10 \text { ş } \\ £ & 2 \text { ş } \\ £ & 2 \text { ş } \\ £ & 4 \text { ş } \\ £ & 2 \text { ş } \\ £ & 4 \text { ş } \\ 4 £ & \text { ş }\end{array}$

[ 24 ] Pàg. 76 : «Die 3 Juli 1767»: "Lo organista diu que lo manxador no vol / portar lo simbol sobre que diu se li aumenta / en anys passats 6 lliures de dita oblig / Que los 6 lliures se entreguen al organista / y que los hi entregue de poch en poch. [...] [Pàg. 98 :] Dictus Dominus Canonicus Botines Sincus proposuit / Que lo Organista lo ha informat que lo manxador / [Pàg. 99 :] no vol aportar lo simbol als Llochs ahont hi ha funcio de / Capella; i com sa Merce tinga intelligencia, que / se aumenta a dit manxador lo salari de sis lliuras / annuals ab la dita obligacio si apar a V.S. podria / servirse resoldrer que las ditas sis lliuras las / cobria lo organista, y que cada vegada, que haura de / de portar lo simbol lo manxador, li done alguna cosa / de ditas sis lliuras, y si finit lo any sobra alguna / cosa que tambe li entregui. En Dms Caps de Placet"

[ 25 ] Pàg. 101 : «Die 6 Juli 1767»: "Dictus Domenicus Can ${ }^{\text {cus }}$ Botines Sindicus pro / possuit que lo Com ${ }^{1}$ Monlleó de esta Ygla se tro- / ba ab Carta del Me de Capella de $\mathrm{S}^{\text {ta }}$ Maria / de Barcelona en la que li diu que y ha un pre / tensor a una de las sustentorias de esta Ygla, y / lo avisa de las circumstancias de dit Pretensor / [Pàg. 102 :] qual carta si V.S gusta se llegira Domini legatur / qua lecta Domini Capitulantes determinarunt $\mathrm{q}^{\mathrm{e}}$ / se envie copia de dita carta al $\mathrm{S}^{\text {or }} \mathrm{D}^{\mathrm{n}}$ Joan Ri- / bas pera que quan dit Pretensor compareguia / en presencia sua (Lo que podra dit $\mathrm{Com}^{1}$ monlleó / escriurer a dit Mestre de Capella ho diga a / dit Pretensor), lo fara dit $S^{\text {or }} \mathrm{D}^{\mathrm{n}}$ Joan probar per / algun subjecte que sia de sa satisfaccio y avisar / que cosa es aixi en veu com en Pericia al cant Pla / y cant figurat".

[ 26 ] Pàg. 111 : «Die 1 Agost 1767»: "Dms Cans Botines Sindicus proposuit, que se ha / rebut Carta per V.S. de $\mathrm{D}^{\mathrm{n}}$ Joan de Ribas sin- / dich de V.S. en Barcelona de data del 28 de / Juliol passat, que si V.S. gusta se llegira. Domini / legatus qua lecta determinarunt, que los $\mathrm{S}^{\text {ors }}$ Arxivers / se servescan mirar si en lo arxiu se trobaran / altras noticias en punt de la Casa de Barcelona / y se li envien, y que en lo

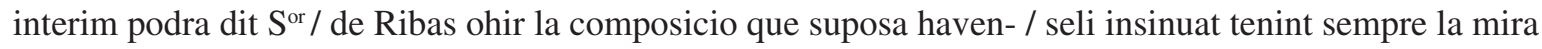
de que / no se impedesca la vista desde dita Casa. Dictus Dms Can ${ }^{\mathrm{s}}$ Botines Sind ${ }^{\mathrm{s}}$ Proposuit, que se / ha rebut altra carta per V.S. del $\mathrm{M}^{\mathrm{t}} \mathrm{Y}^{1 l^{\mathrm{e}}} \mathrm{Cap}^{1}$ de / Vich de 26 de Juliol pasat que si $\gamma$ gusta se / llegira Domini determinarunt que se fase alguna / refleccio sobre lo contengut en dita carta, y que los / $\mathrm{S}^{\text {ors }}$ Arxivers se servescan mirar si se trobaran / documents y adminiculos que pugan coadjuvar al intent / al dit $\mathrm{M}^{\mathrm{t}} \mathrm{Yll}^{\mathrm{e}}$ Cap de Vich, y a son temps respongan a [ Marge : "Dita Causa"] ".

[ 27 ] Pàg. 113 : «Die 13 Augusti 1767»: "Carta de Ribas de 11 del corrent / Que se li digne que se aplique, que en lo temps / podra servirli, y que se li done alguna cosa per lo viatge [ Marge : "dela obra" ] / en quant al sustentor, Que una semana $p^{t}$ al [?] / que los dos a matinas. [...] [Pàg. 115 :] Dictus 
$\mathrm{Dmu}^{\mathrm{s}}$ Can Botines $\operatorname{Sin}^{\mathrm{s}}$ Prop $^{\mathrm{t}}$ Que se ha rebut altra carta per V.S de $\mathrm{D}^{\mathrm{n}}$ Joan de Ribas Sin ${ }^{\text {ch }} /$ de V.S. en $\mathrm{Bar}^{\text {na }}$ de 11 del corrent, que si V.S. gusta / $\mathrm{Dm}^{\mathrm{s}} \mathrm{Can}^{\mathrm{s}}$ Botines dixit, Que lo sub / gecte que volia entrar per Sustentor, del qual / envia los informes dit $\mathrm{S}^{\mathrm{r}} \mathrm{D}^{\mathrm{n}}$ Joan ha com / paregut en esta y atesos estos, y lo que ha / donat lo $\mathrm{M}^{\mathrm{e}}$ de Capella de esta Igla no esta / en disp ${ }^{\circ}$ de admeterse per ara, si sol apar / se li podria donar alguna esperansa de / admeterlo si en lo espay de un any o mes / ell se perfeccionava en lo Cant pla, y pot / apendrer de Cant figurat Et Dmi Cap ${ }^{1}$ Det $^{\mathrm{t}} /$ Placet, y que de la Adm ${ }^{\circ}$ de la obra se li / done alguna cosa per son retorn a disp ${ }^{\circ} /$ del $S .{ }^{\text {or }}$ Obrer y que lo $\mathrm{S}^{\text {rs }}$ Arxivers se / servescan respondrer a dita carta segons / son tenor. Dmu $\mathrm{Can}^{\mathrm{s}}$ Llorens $\mathrm{Sind}^{\mathrm{s}} \mathrm{prop}^{\mathrm{t}}$ que lo Sustentor / nou per mes que se aplique ab gran diffi / cultat podra regir be lo Cor a matinas / sino assiteix lo altre susten / tor Y si apar podria V.S. servirse resoldrer / que en la una semmana se digan matinas / resadas y en la altra cantadas ab assisten / cia dels dos sustentors. Et Dmi Cap ${ }^{\mathrm{s}}$ Det ${ }^{\mathrm{t}}$ Placet".

[ 28 ] Pàg. 117 : «Die 22 Agusti 1767»: "Que se han de donar als sustentor los papers. [Pàg. 118 :] Dictus Dom ${ }^{\mathrm{s}}$ Can $^{\mathrm{s}}$ Botines Sindicus proposuit : / que respecte que lo subjecte que molts Individuos / de V.S. han ohit Cantar en lo Chor apar lo conci / deran molt abil per Sustentor, Se li han demanat los / papers que tenia per veurer si acudint novament / a Sa Santedat obliganse V.S. en ferli las congrua / de 100 rals de vuit annuals fins y atant que / V.S. puga provehirlo de algunas Pessas Eclesiastica / de esta Igla, que tinga las mateixas o major congrua / haventlos examinat Sa Merce si apar no ser molt / dificultos el lograr de Sa Santedat dit Subjecte un / Breu [ Marge : "per poder secularisar ] favorable per poder exereir en esta Igla dit / empleo de Stustentor. Perloque V.S. ha de servir re- / soldre lo de son agrado. Et Domini Capitulantes deter / minarunt, que pnte Memorial a Sa Señoria y se pen- / dra resolucio sobre son contengut".

[ 29 ] Pàg. 119 : «Die 24 Augusti 1767»: [ «Marge : "Que se li asseñali $100 \mathrm{R}^{\mathrm{s}}$ de 82"] "Botines suplica de $\mathrm{D}^{\mathrm{n}}$ Ambros Garcia de los Dos Pbre / $\mathrm{R}^{\mathrm{s}}$ de 8. / Que se anomene Sustentor ab lo salari de $100 \mathrm{R}^{\mathrm{s}}$ de 8 annuals servint a la Igla en lo que puga / y [?] per la sustentoria, com en tocar los instru- / ments que sapia , y fan [?] las Sindichs y / Arxivers per a posar en clar esta resolucio / y que en lo interim residescan ab habits / de Chor. [Pàg. 120 :] Dictus Dms Can ${ }^{\mathrm{s}}$ Botines Sinds Prop / Que D ${ }^{\mathrm{n}}$ Ambros Garcia de los Dos Pbre / li ha entregat una suplica per V.S. que si / gusta [?] $\mathrm{Dm}^{\mathrm{s}} \mathrm{Cap}^{1} \operatorname{Det}^{\mathrm{t}}$ anomenar com en efecte / anomena al mencionat $\mathrm{D}^{\mathrm{n}}$ Ambrós Garcia de los Dos / per un dels sustentors de esta Igla ab lo salari / de cent reals de vuit al any, y demes utils de dis- / tribucions de Chor y Capella que deura gosar ser- / vint en lo mencionat empleo, ab las facultats que / tinga encara que per impediment natural de enfer- / metat o perdua de veu no puga servirlo en lo tot / [Pàg. 121 :] per lo cas que Sa Santedat se digne secularisarlo y / dispensarli los vots de sa proteccio Religiosa ma- / nant a mi lo Votts y Secretts Ynfrit doni testimoni de / est son nombrament al Suplicant per los efectes que / puga serli convenient".

[ 30 ] Pàg. 121 : «Die 4 Septembris 1767»: "Botines sobre el asiento que se deu donar al nou / sustentor : Que lo $\mathrm{S}^{\text {or }}$ Ribas informa / Que se posa al segon chor al costat del sustentor per ara fins a nova disp ${ }^{\circ}$. [...] [Pàg. 122 :] Dmu Can $^{\mathrm{s}}$ Botines Sind ${ }^{\mathrm{s}}$ Prop ${ }^{\mathrm{t}}$ Que se ha / format lo borrador del certificat que se deu / entregar a $\mathrm{D}^{\mathrm{n}}$ Ambros Garcia de los Dos / relatiu a la nominacio feta per V.S. en / sa Persona per altre dels sustentors de esta / Igla a fi de que ab ell puga recorrer a Sa / San ${ }^{t}$ per a que se digne secularisarlo ab / lo salari annual de $100 \mathrm{R}^{\mathrm{s}}$ de 8 y altres adventicis, que / li concedeix V.S. una vegada que Sa / $\mathrm{San}^{\mathrm{t}}$ ha 
manifestat inclinarse a secula- / risarlo segons apar de un escrit de la / Sagrada penitenciaria de 16 agost / 1766 que pnta a V.S que pnt / ab dit certificat se llegira si V.S gusta / Dni legr dit certificat Quo lecto / Despues de saverlo aprobat de $e^{t}$ que se entregue authentich adit $\mathrm{D}^{\mathrm{n}}$ ambros / per mi lo Nott y Seg ${ }^{\mathrm{o}}$ Infraescrit per los / efectes que puga serli convenient. Dnu ${ }^{\mathrm{s}} \mathrm{Can}^{\mathrm{s}}$ Botines Sind ${ }^{\mathrm{s}}$ Prop $^{t}$ Que V.S. / se ha de servir resoldre quin lloch deura / ocupar lo nou sustentor en lo Chor / [Pàg. 123 :] pues segons escriu D Joan de Ribas al $8^{\text {bre }}$ lany / [?] en la Igla de Barna los 4 sustentors / estan assentats en las cadiras superiors ab / alguna distancia entre si y ab preferen / cia a tots los oficials de dit Cap1. Que / lo estar un dels sustentors en lo Chor / alt y lo altre en lo baix, segons lo ban / informar los mateixos sustentors te / un grandisim inconvenient de que no se / ohien, y per consequent de no donar / la Armonia al Chor necessaria y los apar / que fora millor que estassen inmediats / en lo assiento en lo Chor. Per lo que V.S. / se ha de servir resoldre lo de son / para. En $\mathrm{Dm}^{\mathrm{s}} \mathrm{Cap}^{\mathrm{l}} \mathrm{Det}^{\mathrm{t}}$ Que per / ara y fins que se ordene in forma / del nou Plan de la Igla lo modo / ab que deura governarse lo Chor / lo Sustentor nou se pose inmediat / al Sustentor del segon Chor. Y que / respecte que aiximateix se deu espe- / rar lo resultat de dita disposicio [Pàg. 124 :] per lo modo ab que deuhen anar a les / Professons, que vaigen los dos Sustentors / antichs vestits ab capas y Bordons, y / lo modern Sustentor ab un dels Sem- / maners vestits aixi mateix prop dels Sustentors".

[ 31 ] Pàg. 129 : «Die 19 September 1767»: "Botines un sustentor ha servit 5 anys a la Seu / de Urgell, si se admetra ara o despres / que los Sindichs lo proven quant vinga la ocasio, y que se informa $\mathrm{D}^{\mathrm{n}}$ Jaume / Ferrer despido de $\mathrm{D}^{\mathrm{n}}$ Jaume".

[ 32 ] Pàg. 132 : «Die 19 September 1767»: "Dictus Don Cans Botines Sinn propo / suit que pochs dias ha que / comparague un subjecte que ha servit sinch / anys de sustentor en la Igla Cathedral de la / Ceu de Urgell, Y desitjaria entrar en esta Igla / per sustentor : Ab q ${ }^{\text {e }}$.S. se ha de servir resoldrer / lo de son agrado. $\mathrm{Dm}^{\mathrm{s}} \mathrm{Cap}^{\mathrm{s}}$ determ $\mathrm{q}^{\mathrm{e}}$ los $\mathrm{S}^{\text {ors }} \mathrm{Sind}^{\mathrm{s}} / \mathrm{se}$ servescan provarlo y $\mathrm{q}^{\mathrm{e}}$ lo $\mathrm{S}^{\text {or }} \mathrm{d}^{\mathrm{n}}$ Jaume Ferrer / se servesca informarse dels motius ha tingut per / deixar la sustentoria de dita $\mathrm{Cateh}^{1}$, com y de / las qualitats del subgecte".

\section{Any 1777}

[ 33 ] Pàg. 653 : «D. 28 Jan. 1777» : "D Can ${ }^{s}$ Llo. Prop ${ }^{t}$ Que lo s ${ }^{\text {or }}$ Arch $^{\mathrm{e}}$ fa pnt a V.S / un mem[oria] ${ }^{1}$ dels dos succentors, en lo qual demanan / alguna subven ${ }^{\circ}$ pera mantenirse ynterim no / cobrian lo quadrimestre com a Comensals, y per / [Pàg. 654 :] altres motius, qe en dit mem[oria] ${ }^{1}$ van compresos; Lo / qual si gusta $\gamma$ Dm Leg ${ }^{\mathrm{r}}$ Quo lecta: $\mathrm{Dm}^{\mathrm{s}} \mathrm{dix}^{\mathrm{t}} /$ Que los $\mathrm{S}^{\text {ors }} \mathrm{Sind}^{\mathrm{s}}$ exponian al $\mathrm{s}^{\text {or }} \mathrm{Arch}^{\mathrm{e}}$ lo que / fins vuy ha suplert y entregat lo Cap ${ }^{1}$ per acudir / a Sas urgencias als men ${ }^{\text {ts }}$ Susccentors, així com q / desde luego sels poden entregar y sels entrega / ran si sels acomoda las $34 £ \mathrm{q}^{\mathrm{e}}$ percebeixen de la Admº de la obra, ab tot lo demes $\mathrm{q}^{\mathrm{e}}$ los / aparega convenient fer pnt a V.S per a precau / cionar semblants carrechs en lo successiu a la / adm ${ }^{\circ}$ si no obrant $\mathrm{l}^{\mathrm{a}}$ Ynformació, apar el $\mathrm{S}^{\text {or }} \mathrm{Arch}^{\mathrm{e}} \mathrm{q}^{\mathrm{e}}$ deu donarsels algun tant diari o mensual / o $\mathrm{p}^{\mathrm{r}}$ una vegada solam ${ }^{t}$; per a socorrer las sobreditas / urgencias, ho executara lo Cap ${ }^{1}$ de la manera $\mathrm{q}^{\mathrm{e}}$ lo $\mathrm{S}^{\text {or }}$ $\mathrm{Arch}^{\mathrm{e}}$ ho disposia". 
[ 34 ] Pàg. 660 : «D. 4 February 1777» : "D $D^{\mathrm{s}}$ Llor $^{\mathrm{s}}$ Prop $^{\mathrm{t}}$ Que en conseq ${ }^{\mathrm{a}}$ de la comissio feta $\mathrm{p}^{\mathrm{r}}$ V.S / al S ${ }^{\text {or }}$ Ricci y a sa Merce en lo $\mathrm{Cap}^{1}$ de 28 Janer / prop passat [ Margen : "sobre lo recurs / per at Yll ${ }^{\mathrm{m}}$ / $\mathrm{p}^{\mathrm{r}}$ los dos sustentors / Jph Garcia y / Ramon Mirosa" ] se conferiren los dos ab su Yll ${ }^{\mathrm{a}}$ exposantli / lo q $\mathrm{q}^{\mathrm{e}}$ dona de gratificacio a dits dos susttentors, en att ${ }^{\circ} /$ dels gastos $q^{\mathrm{e}}$ havian tingut, com també, tot lo que / percibiran de la Ygta, en lo estat actual de no trobarse / ordenats sacerdots; lo q apar es mes q $\mathrm{q}^{\mathrm{e}}$ suficient $\mathrm{p}^{\mathrm{r}} / \mathrm{sa}$ decent manutencio; $\mathrm{y} \mathrm{q}^{\mathrm{e}}$ si be las distribuicions $\mathrm{q}^{\mathrm{e}} / \mathrm{en}$ virtut del nou plan guañan los Comensals se / pagan $\mathrm{p}^{\mathrm{r}}$ quadrimestre y no entraran a cobrar lo qua / drimestre corrent, fins al primer de Maig, sels pot / subvenir $\mathrm{p}^{\mathrm{r}}$ lo $\mathrm{q}^{\mathrm{e}}$ necessitan fins a dit temps, adelan / tantlos part, o, tot lo salari $\mathrm{q}^{\mathrm{e}}$ dehuen percebir / de la Obra, $q^{e}$ es de $34 £$ a cada hu. Y que / en vista de axo apareixia infundada sa peticio / y quaregue a S.J. molt arreglat; Pero havent / posteriorment parlat S.Y ab sa Mereé pero que / no obstant si a S.J. li apareixia $\mathrm{q}^{\mathrm{e}}$ sels subvingues / en alguna cosa de la $\mathrm{Adm}^{\circ}$ de la obra, V.S / estaria a sa determ ${ }^{\circ}$ en vista del qual / [Pàg. 661 :] respongué S.J. q $q^{\mathrm{e}}$ li apreixian consideradas las / rahons $\mathrm{p}^{\mathrm{r}}$ no donar a dits sustt ${ }^{\text {ors }}$ altra subven ${ }^{\mathrm{o}}$ $q^{\mathrm{e}}$ la de adelantar a cada hu las $34 £$ de la Obra: No / obstant, havent posteriorm ${ }^{t}$ S.J. fet conversacio ab sa / Merce sobre lo mateix, digué, $\mathrm{q}^{\mathrm{e}}$ havent fet refleccio / sobre lo $\mathrm{q}^{\mathrm{e}}$ se li havia exposat en est assumpto, li apareixia $q^{\text {e }}$ sels podria donar a cada hu ames dels ade / lantaments referit $10 £$ de la $\mathrm{Adm}^{\circ}$ de la obra".

[ 35 ] Pàg. 662 : «Dia 10 Feb 1777» : "D" Can ${ }^{s}$ Llorens Prop ${ }^{t}$ Que essent notoria a V.S ta resisiteneia la falta de acistencia del comensal del / segon Chor, aqui se havia avisat pera cantar los versets; a

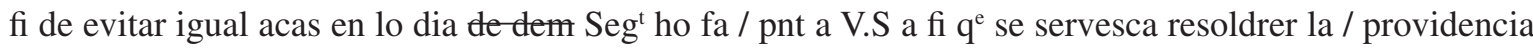
$q^{\mathrm{e}}$ se podra pendrer per a precausionarho. [Pàg. 663 :] Et Dom ${ }^{\mathrm{s}} \operatorname{Det}^{\mathrm{t}}$ Que los $\mathrm{S}^{\text {ors }}$ Sind $^{\mathrm{s}}$ ho comuniquen al $\mathrm{S}^{\text {or }} \mathrm{Arch}^{\mathrm{e}}$ fentli evidencia dels motius $\mathrm{q}^{\mathrm{e}}$ apar / indican ser obliga ${ }^{\circ}$ dels comensals, lo cantar / los versos en aquells dias $\mathrm{q}^{\mathrm{e}}$ antes se anomenaven / de paborders $\mathrm{p}^{\mathrm{r}}$ celebrarser ab alguna solemnitat / $\mathrm{p}^{\mathrm{r}}$ raho de alguna fundacio $\mathrm{q}^{\mathrm{e}}$ un dia no te lloch / $\mathrm{p}^{\mathrm{r}}$ falta de renda; y al mateix temps $\mathrm{q}^{\mathrm{e}}$ se multia lo referit comensal aqui tocava fer lo doble / ab la distrib corresponent a les Vespres".

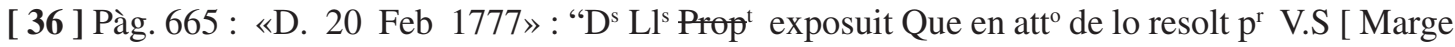
: "en lo rotllo del dia 10"] ab lo / motiu de la falta y resistencia del Com $\mathrm{Col}^{1} \mathrm{deg}$ son Chor / aqui tocava fer lo doble de baixar a tocar los versets / en las vespres de $S^{t}$ Ramon se conferiren sa Merce / y lo $S^{\mathrm{r}} \mathrm{Can}^{\mathrm{e}}$ Ricci ab lo $\mathrm{S}^{\mathrm{r}} \mathrm{Arch}^{\mathrm{e}}$ comunicanli lo / ocorregut a fi de $\mathrm{q}^{\mathrm{e}}$ se dignás S. J providenciar pera $\mathrm{q}^{\mathrm{e}} /$ no succeis lo mateix en lo ofici y vespres del dia seg ${ }^{t} /$ lo q $^{e}$ executá S. J. pasant un recado al Gremi de / Comensals $\mathrm{p}^{\mathrm{r}}$ est fi; Y apareixent molt propi de la / att ${ }^{\circ}$ de V.S lo donar gracias a S. J. $p^{\mathrm{r}}$ esta demostracio, / havent significat

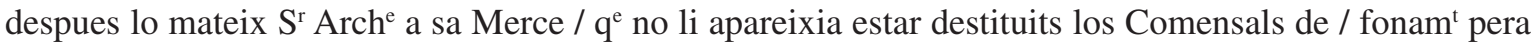
repugnar lo tocar ${ }^{\text {los }}$ versets y antifonas corres- / ponents en dit dia de $S^{t}$ Ramon y altres que antes cele / brava la Iglta amb major solemnitat per alguns motius / $\mathrm{q}^{\mathrm{e}}$ vuy dia no exhisteixen, podra V.S resoldrer lo $\mathrm{q}^{\mathrm{e}} /$ li aparega convenient executar en esta depend ${ }^{\mathrm{a}}$. Et / Dom dett ${ }^{\mathrm{t}} \mathrm{q}^{\mathrm{e}}$ se forma una minuta substancial com/ prehensiva dels motius $q^{\mathrm{e}}$ indican ser de la obligacio dels Comensals lo reputar vuy dia alguns dels dias / $\mathrm{q}$ se anomenavan de Pabordes per purament / dobles, y $\mathrm{p}^{\mathrm{r}}$ conseq $^{\mathrm{t}}$ lo tocar los versets y antifonas, y / fentse pnt dita minuta en lo Cap prop $^{\mathrm{m}}$ vinent, se / determinara lo q aparega convenient comunicar al $\mathrm{S}^{\mathrm{r}} \mathrm{Arch}^{\mathrm{e}}$."

[ 37 ] Pàg. 670 : «D. 3 Mars 1777» : " $\mathrm{D}^{\mathrm{s}} \mathrm{Can}^{\mathrm{s}}$ Vilallonga prop ${ }^{\mathrm{t}}$ Que en virtut de lo / resolt $\mathrm{p}^{\mathrm{r}}$ V.S en lo $\mathrm{Cap}^{1}$ anteriort se ha format / la minuta comprehensiva dels motius $\mathrm{q}^{\mathrm{e}}$ indican / estan obligats los Co- 
mensals fer algun dia $\mathrm{q}^{\mathrm{e}}$ antes / se anomenavan de Pabordes. Lo qual si V.S gusta [?] / Qua lecta: Dom dix ${ }^{\mathrm{t}}$ Que se pase en escrit al / $\mathrm{S}^{\text {or }}$ Arche."

[ 38 ] Pàg. 672 : «D. 10 Marty 1777» : " $D^{s}$ Cann $^{\mathrm{s}}$ Llor $^{\mathrm{s}}$ Sind $^{\mathrm{s}}$ prop $^{\mathrm{t}}$ Que hi ha $\mathrm{p}^{\mathrm{r}}$ V.S un mem[oria] $]^{1 /}$ del $\mathrm{S}^{\mathrm{r}}$ Ambrosio garcia de los Dos, ab una certificacio / del $\mathrm{S}^{\mathrm{r}} \mathrm{Jph}$ Vilarroya a fi de $\mathrm{q}^{\mathrm{e}}$ mediant los motius / $\mathrm{q}^{\mathrm{e}}$ expresa se digna posar un substitut $\mathrm{q}^{\mathrm{e}}$ servesca / son empleo de Sustentor, deixantli a dit Sup ${ }^{\mathrm{t}}$ la / renda $\mathrm{q}^{\mathrm{e}}$ necessita per sa congrua o descent ma- / nutencio: Lo qual $\operatorname{Leg}^{\mathrm{r}} \mathrm{n}^{\mathrm{o}}$ [?] Quo lecto: Dom dix / Que fasca $\mathrm{p}$ [rese]nt aixi las causas de esta solicitut, com lo Certificat qe presenta als $\mathrm{D}^{\mathrm{rs}}$ Pau Palleja y Pere / Joan Sola y $S^{\text {or }}$ salvador Corbella peraq / consultant los tres sobre dit assumpto exposian / [Pàg. 673 :] son dictamen al Cap.",

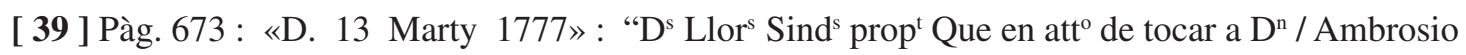
Garcia per la Semmana proxim vinent / y no haven presentat encara lo dictamen dels metges / y Cirurgiá Corbella pera resoldrer V.S sobre sa / pretencio, ha pensat insinuar a algu dels altres sus- / tentors, $\mathrm{q}^{\mathrm{e}}$ su-

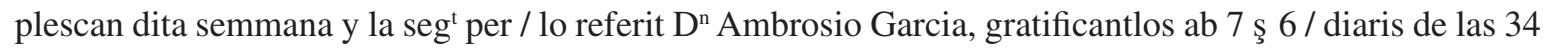
$£ q^{\mathrm{e}}$ percebeix annualment de la Obra / y prevenitlos $\mathrm{q}^{\mathrm{e}}$ no olvidian lo registrar los Llibres / y posarlos a son temps al faristol. Tot lo que executara / si es de la aproba ${ }^{\circ}$ de V.S".

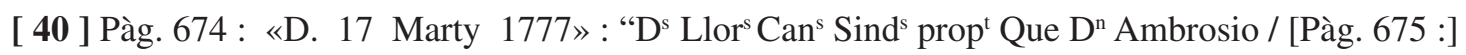
Garcia tenia ya desparats entregats los certificats / dels metges i Cirurgia los quals, si V.S gusta $\gamma \operatorname{leg}^{\mathrm{n}} /$ [?]

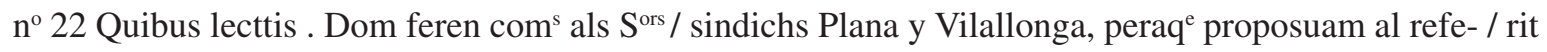
$\mathrm{D}^{\mathrm{n}}$ Antonio lo medi ab q $\mathrm{q}^{\mathrm{e}}$ apar exonerarse de / son empleo, si li acomoda adaptarlo, ab la renda y / precaucions, $\mathrm{q}^{\mathrm{e}}$ se exposaren en la Junta $\mathrm{Cap}^{\mathrm{r}}$ y las / demes, $\mathrm{q}^{\mathrm{e}}$ se oferescan y trobian $\mathrm{p}^{\mathrm{r}}$ convenients insinuarli / dits $S^{\text {ors }}$ comisionats, despues de cuya Diligen ${ }^{a}$ se podrá / fer $\mathrm{p}\left[\right.$ rese]nt al Cap ${ }^{1}$ sa resulta pera deliberar ab / acert lo qe convinga".

[ 41 ] Pàg. 677 : «D. 20 Mars 1777»: " $D^{s}$ Llor $^{s}$ prop $^{t}$ Que se ha tirat la minuta pera / comutar ab lo empleo de Organista Supernumerari / la de plasa de Sustentor, qe ocupa D ${ }^{\text {n }}$ Ambrosio Gar- / cia, y apar, no poder desempeñar dificultar son desem- / peño las Certifica ${ }^{\mathrm{s}}$ p[rese]ntadas; Lo qual si V.S gusta se / llegira: Dom leg ${ }^{\mathrm{r}}$ Qua lecta: Dom dix ${ }^{\mathrm{t}} \mathrm{q}^{\mathrm{e}}$ antes de / entregar copia de las clausulas convenients peraque / puga deliberar lo men ${ }^{t}$ Garcia sobre la proposicio / se fasia $\mathrm{p}[\mathrm{rese}] \mathrm{nt}$ esta depend $\mathrm{d}^{\mathrm{a}} \mathrm{al} \mathrm{s}^{\text {or }} \mathrm{Arch}^{\mathrm{e}}$ afi de / veurer si mereix sa aprobacio. $D^{s}$ Llor $^{s}$ prop $^{t}$ Que haventli demanat Jph Domingo si podria residir al Chor ab Sobrepellis y Museta / o bé ab Sobrepellis tantsolament; no obstant de ofe- / rinseli lo no tenir la qualitat de ser oficial del / Cap ${ }^{1}$ li ha aparegut deixar esta deliberacio per / V.S. Et Dom dix ${ }^{t}$ Que residesca sens Museta / com los demes [Pàg. 678 :] Ben[efici]ats de la Igla. D Can $^{\mathrm{s}}$ Palomar Adm ${ }^{\text {or }}$ opere prop ${ }^{\mathrm{t}}$ Que hi ha / $\mathrm{p}^{\mathrm{r}}$ V.S un mem[oria] $]^{1}$ de Pau Jorda en lo $q^{\mathrm{e}}$ demana / se digna V.S augmentarli lo salari de $35 £ /$ annuals q $\mathrm{q}^{\mathrm{e}}$ te per lo empleo de primera trompa; / Lo qual $\gamma n^{\circ} 27$ Quo lecto: Dni dix ${ }^{t}$ Que los mateixos / $\mathrm{S}^{\text {ors }} \mathrm{Can}^{\mathrm{s}} \mathrm{Comi}^{-}$ sionats en lo Cap enteced $^{t} \mathrm{p}^{\mathrm{r}}$ la depend ${ }^{\mathrm{a}}$ / dels Monjos Vbia y Ripoll conferencien y reflexio- / nen sobre est assumpto, reportant a son temps son / dictamen a sa Señ ${ }^{\mathrm{a}}$ pera deliberar lo $\mathrm{q}^{\mathrm{e}}$ aparega / convenient."

[ 42 ] Pàg. 679: «D. 2 Ap $1777 »: ~ " D{ }^{s} C^{s}{ }^{s}$ Ricci propt Que hi ha un mem de / D Ambrosio Garcia, ab lo qual respon ala prop ${ }^{\circ} \mathrm{q}^{\mathrm{e}}$ se li feu per part de V.S pera substituirli altre / sustentor en son lloch; lo qual si V.S gusta $\gamma n^{\circ} 30 /$ Quo lecto: Dom dix ${ }^{t}$ Que los mateixos $S^{\text {ors }}$ Comisionats / refleccionen esta 
mateixa, y veijan lo millor / modo ab q e podrá providenciarse esta depend / comunicantho al $\mathrm{S}^{\text {or }} \mathrm{Arch}^{\mathrm{e}}$ si los apar[ega] convenient. [...] [Pàg. 680 :] $\mathrm{D}^{\mathrm{s}} \mathrm{Can}^{\mathrm{s}}$ Palomar prop ${ }^{t}$ Que haventse concluhit a / satisfo sua y del $\mathrm{M}^{\mathrm{e}}$ de Capella $\mathrm{p}^{\mathrm{r}}$ lo punt la / Campana de tocar a Morts, y havent significat / lo mateix Campaner $\mathrm{q}^{\mathrm{e}}$ li ocasionava admiracio / de veurer lo puesto ahont estava collocada esta ylas / demes Campanas, quals, li apareixia estarian molt / millor posadas als ventanals, si era factible execu- / tarho sens perfidia de la obra, y havent adquirit / noticia així per medi del $\mathrm{M}^{\mathrm{e}} \mathrm{Jph}$ Gil, com de $\mathrm{M}^{\mathrm{e}}$ / Jordi Miralles q e podia executarse dita obra sens perjudici del Campanar ni perill de las Campanas / y no ab gran cost, li ha aparegut ferho pnt a V.S / a fi de $q^{\mathrm{e}}$ se servesca determinar si la pusaran / y collocaran al mateix puesto $\mathrm{q}^{\mathrm{e}}$ antes estava o be / al finestral corresponent a ell. Et Dom dix ${ }^{t}$ [Pàg. 681 :] q q $^{\mathrm{e}} \mathrm{S}^{\mathrm{r}}$ Obrer y lo $\mathrm{S}^{\mathrm{r}}$ Vilallonga fasen examinar / radicalm ${ }^{t}$ per los referits $\mathrm{M}^{\mathrm{e}}$ Gil y Miralles / agreganthi $\mathrm{M}^{\mathrm{e}} \mathrm{Jph}$ Carafí y $\mathrm{M}^{\mathrm{e}}$ Carlos More- / ra tota la obra del Campanar, desde los fonam ${ }^{\text {ts }} /$ exposantlos tots los reparos $\mathrm{q}^{\mathrm{e}}$ sels oferescan, afi de / $\mathrm{q}^{\mathrm{e}}$ refleccionats $\mathrm{p}^{\mathrm{r}}$ dits $\mathrm{M}^{\mathrm{es}} \mathrm{y}$ vista la Obra donan son / dictamen del qual, si resulta no havenhi perill / algun, ni detriment de dita obra del campanar / y plena seguretat en la firmesa del campanar / atesas totas las circunstan $\operatorname{tant}^{\mathrm{s}}$ de la qualitat de / la pedra, com de la forma, Archs y demes, $q^{\mathrm{e}}$ compren / esta, se posia al ventanal q $\mathrm{q}^{\mathrm{e}}$ li correspon la Cam- / pana nova de tocar a morts, y se respontia al / $\mathrm{Cap}^{1}$ lo dictamen dels $\mathrm{M}^{\text {es }}$ pera pendrer sobre / ell la determ ${ }^{\circ} \mathrm{q}^{\mathrm{e}}$ se tinga $\mathrm{p}^{\mathrm{r}}$ convenient. Et Dnus Can ${ }^{\mathrm{s}}$ Ferrer, simul, cum Dno canonico / Nolla dix ${ }^{\mathrm{t}}$ Que protestavan a dita resol ${ }^{\circ}$ per no ser los deu vocals congregats en la junta / la major part del Cap ${ }^{1}$, com los apar $\mathrm{q}^{\mathrm{e}}$ demana / o requireix un negoci de semblant importancia / a cuya propa ${ }^{\mathrm{a}}$. Dom dix ${ }^{\mathrm{t}}$ Que sens obrar esta / se executas lo resolt."

[ 43 ] Pàg. 683 : «D. 8 Abril 1777» : "D"Can Ricci Sind" prop ${ }^{\mathrm{t}}$ Que los $\mathrm{S}^{\text {ors }}$ Canonges / Comisionats havian format una minuta de la renda / $\mathrm{q}^{\mathrm{e}}$ anualmen ${ }^{\mathrm{t}}$ ha significat $\mathrm{D}^{\mathrm{n}}$ Ambrosio Garcia / que cediria $\mathrm{i}$ consignaria a favor de aquell sub- / gecte $\mathrm{q}^{\mathrm{e}}$ V.S anomenas $\mathrm{p}^{\text {ra }}$ substituir son empleo / la qual si V.S gusta

Dni leg n 33 Qua lecta / Dom dix ${ }^{t}$ Que se acceptia aixi com esta en / to paper contingut en dit paper y $\mathrm{q}^{\mathrm{e}}$ comuniquian / lo estat de esta depen ${ }^{\mathrm{a}}$ al $\mathrm{S}^{\mathrm{r}} \mathrm{Arch}^{\mathrm{e}}$ los $\mathrm{S}^{\text {ors }} \mathrm{Can}^{\mathrm{s}} /$ Comisionats procurant $\mathrm{q}^{\mathrm{e}}$ S.J aderesca $\mathrm{a} \mathrm{q}^{\mathrm{e}}$ lo / substitut anomenat y sobredit puga guañar / residint personalm ${ }^{\mathrm{t}}$ aquellas faltas $\mathrm{q}^{\mathrm{e}}$ cometia $/ \mathrm{D}^{\mathrm{n}}$ Ambrosio a las horas Canonicas Anivs / y demes fund ${ }^{\mathrm{s}}$ de la Igla. Per lo q e respecta als Caps Pasquals si be $/ \mathrm{q}^{\mathrm{e}}$ se feren $\mathrm{p}$ [rese]nts algunas especies concernents a ells / se diferi lo pendrer resol ${ }^{\circ}$ alguna."

[ 44 ] Pàg. 685 : «D. $14 \mathrm{Ab}^{1}$ 1777» : " $\mathrm{D}^{\mathrm{s}} \mathrm{Can}^{\mathrm{s}}$ Llor $^{\mathrm{s}} \operatorname{prop}^{\mathrm{t}}$ Que en virtut de lo resolt / en lo Rotllo vingut lo dia 22 dels corrs embia / sa merce a Sal ${ }^{\text {or }}$ Mar $^{\text {co }}$ a fer pnt at / comunicar en escrits $\mathrm{d}^{\mathrm{a}}$ resol $^{\circ}$ als 4 Sust $^{\text {ors }} /$ Salvat, Vila + Garcia y Mirosa, lo q executá aben ${ }^{6}$ tats [Pàg. 686 :] excepto al Jph Garcia per trobarse ausent; peró de / resulta de aso han succeit alguns lances ab dit su (segons / han informat a Sa Mercé los

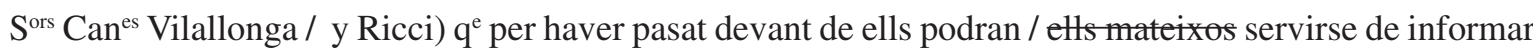
a V.S. Et Dom dix $x^{t}$ placet: en vista del qual lo $S^{\text {or }} \mathrm{Can}^{\mathrm{e}}$ Vilallonga / digue: Que lo mateix disapte dia 12 al vespre acudi / a casa sua Gaspar Salvat dientli $\mathrm{q}^{\mathrm{e}}$ haventseli / comunicat la provid ${ }^{\mathrm{a}}$ de V.S se oferi lo trobarse ab mo / tiu de un Ri devant de la Seu, ab los 3 Susttentors / Vila, Garcia y Mirosa, y considerant q per culpa / sta de ells de no voler servir $\mathrm{p}^{\mathrm{r}} \mathrm{D}^{\mathrm{n}}$ Ambrosio Garcia, havia estat / precis a V.S lo pendrer aquella

6 El manuscrit sobreposa "ab" a l'original "en". 
provid $^{\text {a }}$ no pogue / ell contenirse de envestirlos, queixantse fortam ${ }^{t}$ de / ells, y ab paraulas asperas de la mala correpond ${ }^{\mathrm{a}}$ / ab son Company Sust ${ }^{\text {or }} \mathrm{D}^{\mathrm{n}}$ Ambrosio Garcia, de sa poca aplicacio per lo serveis de la Igla [ Margen : "y son desagra-/himent”] a la qual / devian estar agraits despres de haver rebut molts / particulars beneficis, ab algunas expresions en las / $\mathrm{q}^{\mathrm{e}}$ coneixia haverse excedit mogut unicam ${ }^{\mathrm{t}} \mathrm{del}$ zel / per lo serveis de la Igla; Lo q e posava en sa noticia / esperant $q^{\mathrm{e}} \mathrm{Sa}$ merce vindria a bé de disculparlo / en lo cas de haverhi algun recurs contra ell; a q / respongué Sa Merce $\mathrm{q}^{\mathrm{e}}$ coneixia segons las expre- / sions $\mathrm{q}^{\mathrm{e}}$ dit Salvat li declará qe realm hi havia / hagut Indiscrecio y algun exces; peró qe també ee / ne per raho de haver succehit en un Lloch pub $^{\mathrm{h}} /$ pero $\mathrm{q}^{\mathrm{e}}$ tambe coneixia $\mathrm{q}^{\mathrm{e}}$ totas las ditas expresions / las mereixian molt be los dits 3 susttentors per ser / cert haver ells procehit ab molt desagrahim ${ }^{t} \mathrm{y}$ donat / mostras de sa poca aplicacio; y que $\mathrm{p}^{\mathrm{r}}$ lo tant podia en / esta part quedar descansat: Que despres en la / mateixa vellada comparegue a Casa de Sa Merce / lo sustt ${ }^{\text {or }}$ Jph Garcia, fent forta queixa del / [Pàg. 687 :] mal tracte y paraules asperas q $\mathrm{q}^{\mathrm{e}}$ publicam ${ }^{\mathrm{t}}$ havia usat / contra ell y contra los susttentors Vila y Mirosa lo dit / Gaspar Salvat; a q e respongué Sa Merce trobarse / ya enterat de lo qe havia ocorregut en dit lance, y / q e si bé Sa Merce reconeixia la Indiscrecio de / Salvat en estrellar als dits 3 Sustten $^{\text {ors }}$ ab publicitat / pero q e totas las paraulas asperas ab q $\mathrm{q}^{\mathrm{e}}$ los havia tractat / Salvat eran ben merescudas, y q tot lo mateix $\mathrm{q}^{\mathrm{e}}$ ha- / via dit Salvat, ho deya Sa Merce a saber de fos tres / q dits 3 Sust $^{\text {ors }}$ tenian poca aplicacio y amor a la / Igla , $\mathrm{q}^{\mathrm{e}}$ no reconeixian los favors y Beneficis / $q^{\mathrm{e}}$ havian rebut, $\mathrm{q}^{\mathrm{e}}$ no miravan sinó lo Interes y altres / expresions y $\mathrm{q}^{\mathrm{e}}$ procurasen en avant a correspondrer / millor, a fi de $\mathrm{q}^{\mathrm{e}}$ lo Cap no hagues de aplicar la ma. / Y q $\mathrm{q}^{\mathrm{e}}$ asó es lo q pasá ab Sa Merce y de lo q pot in- / formar a V.S. Y seguidam ${ }^{\mathrm{t}}$ lo $\mathrm{S}^{\text {or }} \mathrm{Can}^{\mathrm{e}}$ Ricci digué / q lo vespre del dia 12 acudiren a casa de Sa Merce los 3 susttentors, Vila, Jph Garcia y Mirosa, y que / Vila digue haversels comunicat lo ordre de V.S de / q ells 3 y Gaspar Salvat servisen un dia cada hu / la Susttentoria per la Semana de $D^{n}$ Ambros Garcia / lo q estavan prontes a executar pero $q^{e}$ respecte / $q^{\mathrm{e}}$ Gaspar Salvat $\mathrm{q}^{\mathrm{e}}$ devia fer lo primer dia, havia / explicat $\mathrm{q}^{\mathrm{e}}$ ell no podia fer las Matinas feya $\mathrm{p}$ [rese]nt / Vila, $\mathrm{q}^{\mathrm{e}}$ si Gaspar Salvat $\mathrm{q}^{\mathrm{e}}$ es lo antiq $\mathrm{q}^{\mathrm{y}}$ Jubilat, no volia comensar, $\mathrm{q}^{\mathrm{e}}$ tampoch no corresponia lo comensar / ell dit Vila, y que aixis veiges Sa Merce de dis- / posar com se havia de observar lo torn: tambe / expresa Vila portant la paraula $\mathrm{p}^{\mathrm{r}}$ tots 3 las queixas / $\mathrm{q}^{\mathrm{e}}$ ells tenian contra Gaspar Salvat, per haverlos / tractat malam ${ }^{\mathrm{t}}$ de paraulas

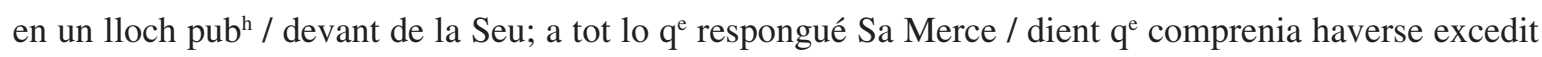
Gaspar Salvat / [Pàg. 688 :] en insultarlos de paraulas en pub ${ }^{\text {h}}$; peró deixant aso / ala part, devian de considerar $\mathrm{q}^{\mathrm{e}}$ la Igla havia de / ser servida, y q e aixis no tenian ells just motiu pera / escusarse de servir $\mathrm{p}^{\mathrm{r}} \mathrm{D}^{\mathrm{n}}$ Ambros Garcia, ni de escusarse / de fer las matinas $\mathrm{p}^{\mathrm{r}}$ salvat, en att ${ }^{\mathrm{o}}$ de $\mathrm{q}^{\mathrm{e}}$ est allegava / impedim y $\mathrm{q}^{\mathrm{e}}$ ell serviria lo dia segt; replica / Sa Merce que no li apareixia conforme al bon / ordre del torn y q no podent comentsar dit Salvat / considerava ser mes propi q comensas Mirosa / com a mes Jove, pues de est modo, tocant lo quant / dia a salvat podria ab en intermedi V.S disposar / $\mathrm{p}^{\mathrm{r}}$ dit dia lo $\mathrm{q}^{\mathrm{e}}$ tingues $\mathrm{p}^{\mathrm{r}}$ convenient; ab lo $\mathrm{q}^{\mathrm{e}}$ se / despedien y sen tornaven dirs 3 sustentors: Peró / $\mathrm{q}^{\mathrm{e}}$ lo dia seg ${ }^{\mathrm{t}}$ al mati de eixir de matinas haventsen / anat Sa merce a despullarse dels habits del Chor en la Capella / $\mathrm{q}^{\mathrm{e}}$ acostuma troba $\mathrm{q}^{\mathrm{e}}$ lo esperava alli lo sut $^{\text {or }}$ Sever / Vila, y li digue a Sa Merce en alta veu devant / de diferents residents, $\mathrm{q}^{\mathrm{e}}$ havian acudit alli a llevarse / los habits de Chor $\mathrm{q}^{\mathrm{e}}$ respecte $\mathrm{q}^{\mathrm{e}}$ lo $\mathrm{S}^{\text {or }} \mathrm{Can}^{\mathrm{e}}$ Llorens / havia embiat la nit antes recado a Ramon Mi- / rosa, $\mathrm{q}^{\mathrm{e}}$ fes matines lo endema y $\mathrm{q}^{\mathrm{e}}$ acudis un / poch antes pera registrar, prevenia y deya dit Vila / 
a Sa Merce $q^{e}$ disposas $q^{e} D^{n}$ Ambros Garcia / anas a registrar los llibres de chor perq e ell no / entenia voler registrar ni registraria dits llibres / a q e respongue Sa Merce $q^{\mathrm{e}}$ una vegada que / lo $\mathrm{S}^{\text {or }}$ Can ${ }^{\mathrm{e}}$ Llorens havia donat disp ${ }^{\circ}$ sobre est / particular, corresponia a sa $a^{\circ}{ }^{\circ}$ no providenciar / cosa mes de parlar ab dit $\mathrm{S}^{\text {or }}$ Llorens, a fi de que / se servis donar aquella disp ${ }^{\circ}$ convenient y / $\mathrm{q}^{\mathrm{e}}$ se faria $\mathrm{p}[\mathrm{rese}] \mathrm{nt}$ en Cap $\mathrm{p}^{1}$ lo $\mathrm{q}^{\mathrm{e}}$ ell deya: Que / [Pàg. 689 :] en lo qe pot informar a V.S en vista de las quals / relacions: Dom dett ${ }^{\mathrm{t}}$ Que los $S^{\text {ors }}$ Sind $^{\mathrm{s}}$ se servescan / donar las providen ${ }^{\mathrm{s}}$ convenients $\mathrm{p}^{\mathrm{r}}$ lo servey de la / Igla, y q al sutt ${ }^{\text {or }} \mathrm{Can}^{\mathrm{e}}$ Ricci altres dels Sinds de V.S / de q ${ }^{\mathrm{e}}$ no volia registrar ni registraria qual exces / se aumenta per haver parlat en altas veus devant / de diferents personas, se li multa en 15 ş y se li / amonesta $\mathrm{q}^{\mathrm{e}}$ done satisfaccio a dit $\mathrm{S}^{\text {or }}$

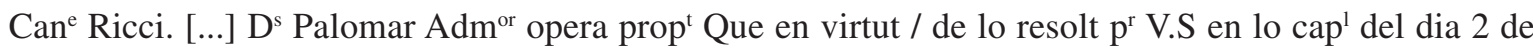
abril / se ha fet la visura del campanar $\mathrm{p}^{\mathrm{r}} 6$ experts, / $4 \mathrm{M}^{\mathrm{es}}$ de cases y 2 fusters, los quals han fet relacio / en deguda forma en poder del Nott de V.S Joan Blay / sobre la firmesa de dit campanar pera sostenir / lo pes de las Campanas, collocadas estas en los Ventanals /sobre la calculacio del pes $\mathrm{q}^{\mathrm{e}}$ soste al pnt la / volta del Campanar entre las Campanas y las pesas / de fusta y y ferro $\mathrm{p}^{\mathrm{r}}$ lo Joch de aquellas y [ Marge : "un calcol separat"] sobre / [Pàg. 690 :] lo import a qe consideran qe deu regularse tot lo / gasto necessari pera collocar ditas Campanas en los / ventanals, en la deguda forma, y ab tota seguretat / al qual efecte sels entrega un paper de preguntas / algunas cathegoricas, y altras generals peraq expliquian / tot lo $\mathrm{q}^{\mathrm{e}}$ tingan $\mathrm{q}^{\mathrm{e}}$ dir de reparos. $\mathrm{q}^{\mathrm{e}}$ sels oferescan / ab tota llibertat; Qual rela ${ }^{\circ}$ [ Marge : "y calcol ab sa addicio"] si V.S gusta $\gamma$ Leg n 37 / Quo Lectas: Dm dett ${ }^{\mathrm{t}}$ Que se Inserte $\mathrm{d}^{\mathrm{a}}$ rel $^{\circ}$ [ Marge : "junt ab lo calcol y sa addicio"] en las / resol $^{\mathrm{s}} \mathrm{Cap}^{\mathrm{rs}}$ y q $\mathrm{q}^{\mathrm{e}}$ respecte de constar de ellas no consi- / derarse ningun perill de collocarse las campanas en los / ventanals, antes be, se esta practica beneficiosa a la / obra del campanar, pues se li alivia de un gran pes / y lo q $\mathrm{e}^{\mathrm{e}}$ li quedara carregara sobre la primera de las / parets; $\mathrm{y} \mathrm{q}^{\mathrm{e}}$ lo cost de esta obra apar moderat $\mathrm{q}^{\mathrm{e}}$ se / execute lo projecte de posar las campanas en los / ventanals y se fase lo ajust ab los mateixos ex- / perts $\mathrm{p}^{\mathrm{r}}$ lo preu $\mathrm{q}^{\mathrm{e}}$ han explicat de $339 £ 4$ ş donantlos / la desferra segons lo calcol ex y addieio ex- / pressat y fent de dit import la rebaixa de las $158 £$ ş eorresponent verificantse / lo q ${ }^{\mathrm{e}}$ se expressa en la addicio de d calcol."

[ 45 ] Pàg. 693 : «D. 26. Ab. 1777» : "D $D^{\mathrm{s}}$ Llor $^{\mathrm{s}}$ prop $^{\mathrm{t}}$ Que ahir dia de $\mathrm{S}^{\mathrm{t}}$ manel no acudi lo / Com Amill al Chor pera cantar las Lletanias, lo q / prevehent Sa Merce previngue ab temps a $\mathrm{M}^{\mathrm{n}}$ Mar- / torell pera suplir en son lloch, lo q espera sera de la / aprob ${ }^{\mathrm{e}}$ de V.S. Y q $\mathrm{q}^{\mathrm{e}}$ així mateix lo referit $\mathrm{Par}^{1}$ ab lo / pretex de recullir los bolletins del cumplim ${ }^{t}$ de la $\mathrm{Par}^{\mathrm{a}}$ / ha faltat com V.S haura reparat a Insensar a vespres / en tots los dias dobles de esta semana; per qual motiu su- / pli p ell lo R Andreu Ripoll; Y havent Sa Merce / preguntat a est si dit $\operatorname{Par}^{1}$ Amill li havia recomanat / est encarrech, hi ha respost $q^{\mathrm{e}}$ no tot lo $\mathrm{q}^{\mathrm{e}}$ fa pnt a / V.S per si apar de multar al referit Par ${ }^{1}$ Amill / $\mathrm{p}^{\mathrm{r}}$ estas faltas: Y com segons se ha experimentat est / Par fa $^{1}$ poch cas de las multas $\mathrm{p}^{\mathrm{r}}$ ser modicas [ Marge : "y ni de la pro / videneia declaracio que feu / V.S de quedar / ell afecte a tots / los carrechs pro- / pis dels parro- / quials"] deura V.S / servirse determinar si se pendrá altra provid ${ }^{\mathrm{a}}$. Et / Dom Cap ${ }^{\mathrm{s}}$ dett $^{\mathrm{t}}$ Que se multe lo Parl Amill en / $^{1} 39$ ş per la falta a cantar las Lletanias. Y en 1 ş per / cada falta a Insensar aplicadoras estas multas als / $q^{\mathrm{e}}$ han suplert, y $\mathrm{q}^{\mathrm{e}}$ se pase un ofici a V. Yll $^{\mathrm{a}}$ po- / sant en sa noticia lo ocorregut, y suplicant se servesca / mant pendrer las providen ${ }^{\mathrm{s}}$ convenients perq $^{\mathrm{e}}$ en / avant sia $\mathrm{d}^{\mathrm{t}}$ Par $^{\mathrm{l}}$ mes exacto en lo cumplim ${ }^{\mathrm{t}} \mathrm{de} / \mathrm{sas}$ obliga ${ }^{\mathrm{s}} . "$ 
[ 46 ] Pàg. 696 : «D. 9. May 1777» : "D Llorens: Que de resulta del offici qe se passa / de part de V.S al Sr Arche sobre las faltas del / Par $^{1}$ Amill se servi sa Ylla insinuar $q^{\mathrm{e}}$ havia / ya donat providencia perq dit Parrâl cum- / plis en lo succesiu ã sa obligacio; pero no / obstant asso pot haver reparat V.S. la falta / que feu lo dia de $S^{\text {ta }}$ creu en no assistir â / entonar las lletanias $q^{\text {e }}$ se cantan en la Pro- / feso de benediccio del terme, per qual motiu / [Pàg. 697 :] disposa sa merce que suplis per ell lo sus- / tentor D ${ }^{\text {n }}$ Ambros Garcia, lo q e fa pnt a V. S / per sa determin ${ }^{\circ}$ Dni determ ${ }^{t}$, que per / esta falta se multe al referit Amill en / £ 39 ş de la subvencio que reb de la satis sacris- / tia, y que se done esta can ${ }^{\mathrm{t}} \mathrm{a}$ dn susten $\mathrm{d}^{\mathrm{n}}$ / Ambros Garcia."

[ 47 ] Pàg. 703 : «d. 28 May 1777» : " $\mathrm{D}^{\mathrm{s}} \mathrm{Can}^{\mathrm{s}}$ Palomar prop ${ }^{t}$ Que Magi canals / libreter li ha entregat un compte de cabals y mans / q diu haver empleat en la recomposicio de 15 / [Pàg. 704 :] Llibres de Chor; qual compte ha aparegut a Sa Merce / ser molt excessiu; y en efecte ha trobat ser aixis $\mathrm{p}^{\mathrm{r}}$ los / Informes $\mathrm{q}^{\mathrm{e}}$ ha pres del altre llibreter, tant $\mathrm{p}^{\mathrm{r}}$ lo que / mira als Cabáls, com p $\mathrm{p}^{\mathrm{r}}$ lo q e mira a las mans, o treball / ło per qual motiu no ha volgut satisferlo sens ferho / pnt com ho executa a V.S p $p^{r}$ la dett ${ }^{\circ}$. Et Dom de / Que veigia Sa Meree dit $\mathrm{S}^{\text {or }} \mathrm{Adm}^{\text {or }}$ de ferli rebaixar dit compte / y si es menester ferlo taxar, y lo pague segons sa dis- / crecio; y tenint pnt lo $q^{\text {e }}$ alguns oficials de la Ygla / abultan sos comptes podra Sameree dit $S^{\text {or }} \hat{o}$ altre $S^{\text {or }} \mathrm{Adm}^{\text {or }}$ en altra ocasio $\mathrm{q}^{\mathrm{e}}$ se ofe- / resca algun gasto servirse de ajustarlo antes per no / exposarse a disputar despres de feta la obra."

[ 48 ] Pàg. 705 : «D. 7 Juny 1777» : "D $D^{s}$ Llo $^{\mathrm{s}}$ prop ${ }^{\mathrm{t}}$ Que hi ha un mem $\mathrm{p}^{\mathrm{r}}$ V.S de Jht / Garcia Susttentor demanant ab motiu de falta de salut / (de $\mathrm{q}^{\mathrm{e}}$ inclou Certificat de Metge) lo permis pera pasar / a Lleyda a posarse en remeys. Qual mem ${ }^{1}$ y $\operatorname{Certif}^{t} \gamma / n^{0} 52$ Quibus lectis. Dni dett': Que no expresant lo Certif $^{\mathrm{t}}$ / necessitat de mudar de aires, ni considerarse motiu just / $\mathrm{q}^{\mathrm{e}}$ lo impedesca de pendrer los remeys en Tarrag ${ }^{\mathrm{a}}$, no / consideran tenir arbitre pera concedirli la p[rese]ncia estant / ausent de la Ciu ${ }^{\mathrm{t}}$, peró q $\mathrm{q}^{\mathrm{e}}$ prenent los remeys en tarra ${ }^{a}$ / se li fa done la $\mathrm{p}$ [rese]ncia en los dias y horas q estiga / impedit de residir. $\mathrm{D}^{\mathrm{s}}$ Llo $^{\mathrm{s}}$ prop $^{\mathrm{t}}$ Que trobantse lo Susttentor $\mathrm{D}^{\mathrm{n}}$ Ambros Garcia / en lo estat $\mathrm{q}^{\mathrm{e}}$ en varias representacions ha expresat de / no poder traurer la veu, y lo Sustt ${ }^{\text {or }} \mathrm{Jht}$ Garcia exposat / ab motiu de sa Indisposicio, a fer algun deix no poder / residir alguns dias, queda lo Chor ab dos sols Comensals / Susttentors, $\mathrm{q}^{\mathrm{e}}$ son Sever Vila y Ramón Mirosa, / lo primer dels quals se experimenta, qe trat esforsa / molt poch la veu ab motiu de ser trencat, per lo / $\mathrm{q}^{\mathrm{e}}$ V.S deu servirse pendrer alguna determ ${ }^{\circ}$ per lo / servey del Chor. Et Domi Cap $\operatorname{dett}^{\mathrm{t}}$ Que se / amoneste al Susttentor Sever Vila q e procure / [Pàg. 706 :] esforsar un poch la veu, pr lo qual no se considera ser / impedimt to trobarse treneat y com no sia fer una forma / extraordinaria no se considera ser impedim ${ }^{t}$ lo trobarse / romput, pues en altra manera seria precis al $\mathrm{Cap}^{1} \mathrm{p}^{\mathrm{r}} /$ lo ${ }^{\text {bon }}$ servey de la Igla pendrer altra providencia, q $q^{\mathrm{e}}$ to / seria mes gravosa. [...] $\mathrm{D}^{\mathrm{s}} \mathrm{Llo}^{\mathrm{s}}$ prop ${ }^{\mathrm{t}}$ Que en la Misa del delelatustro $\mathrm{q}^{\mathrm{e}}$ se / canta a las 7 del mati dels disaptes en la Capella de / $n^{\text {a }} \mathrm{S}^{\text {ra }}$ del Claustro y los dijous en la del Sacrament / se experimenta de algun temps ha $\mathrm{q}^{\mathrm{e}}$ faltan diferents / Mussichs de la Capella, lo $\mathrm{q}^{\mathrm{e}}$ es motiu que esta funcio / causa menos devocio añadintse $q^{\mathrm{e}}$ la propina de cada / hu se entrega durant la misa, lo que i no pot deixar de causar distraccions / y ames se experimenta observa, $\mathrm{q}^{\mathrm{e}}$ luego de cantat lo post comunio / sen van tots sens esperar $q^{\mathrm{e}}$ se acabe la Misa. / Lo q fa pnt a V.s a fi que se servesca a providenciar / lo $\mathrm{q}^{\mathrm{e}}$ regonega ser convenient. Et Dom Cap dett $^{t}$ / que se done ordre al $\mathrm{q}^{\mathrm{e}}$ cuida de cobrar y pagar als Mussichs, $\mathrm{q}^{\mathrm{e}}$ no entregue las propinas fins $\mathrm{q}^{\mathrm{e}}$ sia / acabada la misa y se encarregue a tots los / Mussichs la acistencia y gravedat qedemanan / [Pàg. 707 :] estas funcions." 
[ 49 ] Pàg. 708 : «Die 21 Juny 1777» : "D Llos prop ${ }^{\mathrm{t}}$ Que hi ha un mem $\mathrm{p}^{\mathrm{r}}$ V.S de / Sever Vila Sustt ${ }^{\text {or }}$, ab q exposa la imposibilitat ab q $q^{\mathrm{e}}$ se troba de poder esforsar la veu [ Marge : "ab motiu de trobarse / trencat de lo qe pnta / certificat de 3 Cirurgi / ans, y en consequen / de cumplir a la / amonestacio q ${ }^{\mathrm{e}}$ se / li feu" ] eom se / li amonestá en virtut de resolo de V.S y / q e desitjant curar de son mal, qe fi de treneadura / [Pàg. 709 :] q li produeix dit impedimt de la quat ha resolt en / sent delagrado de V.S de parar a Madrit respecte / de haverse donat noticia en la Gaseta de trobarse / alli un Subjecte $q^{\mathrm{e}}$ ofereix curar perfectam ${ }^{\mathrm{t}}$ de tota / especie de trencadura, lo q posara en execucio si V.S / se serveix donarli lo permis, y ferlo pnt per esta justa / Causa a las distribut ${ }^{\circ}$ Qual mem Si $\gamma n^{\circ} 46$ Quo lecto / Dni dett ${ }^{t}$ Que se li concedesca la llicencia y pncia $\mathrm{q}^{\mathrm{e}} /$ solicita ab la Condicio $\mathrm{q}^{\mathrm{e}}$ dega deixar encarregat a / algu lo suplir $\mathrm{p}^{\mathrm{r}}$ ell ; y aixi mateix dett ${ }^{\mathrm{t}}$ Que se diga / a Gaspar Salvat $q^{\mathrm{e}}$ ajude en lo $\mathrm{q}^{\mathrm{e}}$ puga al sustten ${ }^{\text {ors }} / \mathrm{q}^{\mathrm{e}}$ estigan Instr necessiten de instruccio. [...] $\mathrm{D}^{\mathrm{s}} \mathrm{Llo}^{\mathrm{s}}$ prop $^{t}$ Que hi ha un altre mem ${ }^{1}$ de Jht / Garcia Sustt en qe exposa $\mathrm{q}^{\mathrm{e}}$ haventse posat en / Cura, y necessitant $\mathrm{p}^{\mathrm{r}}$ ella de $\mathrm{e}^{\mathrm{fer}}$ un gasto extraordinari / lo temps $\mathrm{q}^{\mathrm{e}}$ durara, experimenta $\mathrm{q}^{\mathrm{e}}$ no sufraga lo $\mathrm{q}^{\mathrm{e}}$ guaña en la Ygla: en vista del qual demana / a V.S : Que se servesca concedirli una suvencio / [ Marge : "per lo temps / que durara la cura que supo- / san ser cosa / de uns 40 dias "] Qual $\mathrm{m}^{1} \gamma \mathrm{n}^{0} 58$ Quo lecto: Dni dett Que se li / donen 6 de la obra 6 ş diaris durant la cura qe-suposan / ser cosa de uns 40 dies."

[ 50 ] Pàg. 721 : «D. 6 Ag g $^{t}$ 1777»: "Ds $\mathrm{Can}^{\mathrm{s}}$ Vilallonga prop ${ }^{\mathrm{t}}$ Que tenint pnt / lo q $\mathrm{q}^{\mathrm{e}}$ V.S havia disposat en alguns Caps antece- / dents pera posar un Substitut $f$ al Sus ${ }^{\text {or }} / \mathrm{D}^{\mathrm{n}}$ Ambrosio Garcia, havia escrit a Ysidro / Vagueria altre dels ultims opositors per veurer / si se li acomodaria est empleo ab las cir/ cunstancias q V.S se resolt de tenir $140 £$ / de salari annual y las mateixas obligacions / [Pàg. 722 :] q qualsevol altre dels Sust ${ }^{\text {ors }}$. Y haventli res- / post afirmativam ${ }^{\mathrm{t}}$, ho fa pnt ab la mateixa / Carta o resp ${ }^{\mathrm{a}}$ del du Vagueria, la qual $\gamma \mathrm{n}^{\mathrm{o}} 71$ / Quo lecta: Dom annomenaren $\mathrm{p}^{\mathrm{r}}$ substitut de $\mathrm{D}^{\mathrm{n}} /$ Ambrosio al ref $^{\mathrm{t}}$ Ysidro Vagueria durant $\mathrm{t}^{\mathrm{e}}$ beneplacito ab lo referit salari de $140 £$ annuals / las $\mathrm{q}^{\mathrm{e}}$ deura pagarlashi proporcionalm

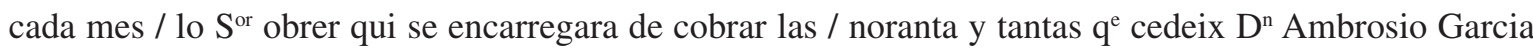
/ en lo paper otorgat en poder del Not Blay / insert en lo Llib. de resolu Cap $^{\text {ars }}$ baix lo dia / 8 de abril de 1777 , y lo no 33 per los rams / q en ell se expresan."

[ 51 ] Pàg. 726 : «D. $27 \mathrm{Ag}^{\mathrm{t}}$ 1777» : " $\mathrm{D}^{\mathrm{s}} \mathrm{Can}^{\mathrm{s}} \mathrm{Llo}^{\mathrm{s}}$ prop $^{\mathrm{t}}$ Que en att ${ }^{\circ}$ de haver disposat / V.S resoldrer despres de la octava de la Assumpta lo / modo y dia en $\mathrm{q}^{\mathrm{e}}$ celebraria publicam ${ }^{\mathrm{y}} \mathrm{y}$ ab tota solem- / nitat las rogativas $\mathrm{p}^{\mathrm{r}}$ lo felis part de la $\mathrm{S}^{\mathrm{ra}}$ Prin- / sesa de Asturias si li apar ocasio oportuna pot / resoldrerho conforme li aparega convenient. Et Dom dix ${ }^{t}$ Que lo diumenge proxim segt se / diga lo ofici a vuit: ab tota Que se avisia al Ajunt. / Que se exposia a tercio lo SS ${ }^{m}$ Sacram ${ }^{t}$. Que / finida la misa pujia lo Chor al Presbiteri a / cantar las lletanias y se reservia more solito. [Pàg. 727 :] D $^{\mathrm{s}}$ Can $^{\mathrm{s}}$ Palomar Ad ${ }^{\mathrm{r}}$ opere prop Que / D ${ }^{\mathrm{n}}$ Ambrosio Garcia presenta a V.S un mem[oria] / en lo $\mathrm{q}^{\mathrm{e}}$ demana alguna subvencio attenent als / gastos y motius q expresa: Lo qual si V.S gusta / se llegira: Dm leg ${ }^{\mathrm{r}}$ Quo lecto Dni dix / Que se li retorni lo mem[oria] dientli q $\mathrm{q}^{\mathrm{e}}$ no telloch / sa peticio y no se inserta $\mathrm{p}^{\mathrm{r}}$ contenir algunas $\mathrm{clau}^{\mathrm{s}} / \mathrm{q}^{\mathrm{e}}$ no conforman ab lo ocorregut antecedent. $\mathrm{D}^{\mathrm{s}}$ Can $^{\mathrm{s}}$ Palomar prop $^{\mathrm{t}}$ Que en virtut de la comisio feta dias atras al $\mathrm{S}^{\mathrm{r}}$ Vilallonga, y a / sa merce per arreglar los salaris dels mu- / sichs, y no admetrer mes suplicas en lo es- / devenidor per auments de salaris: sobre lo / estas del que paga vuy la obra per raho de / dits salaris, han format lo arreglo que si / V.S gusta se llegirá. Dni legert , Quo lecto / dixerunt diferatun." 
[ 52 ] Pàg. 728 : «D. $27^{\text {e }}$ 1777» : "D ${ }^{\text {s }}$ Can $^{\mathrm{s}}$ Palomar Admr opere prop ${ }^{\mathrm{t}}$ Que hi ha un mem[oria] $]^{1}$ del $\mathrm{M}^{\mathrm{e}}$ de Capella en lo qual demana $\mathrm{q}^{\mathrm{e}}$ li sian / resarsidas las $7 £ 13 \mathrm{~s}_{\text {s }} \mathrm{q}^{\mathrm{e}}$ bestragué lo any passat $\mathrm{p}^{\mathrm{r}}$ lo / augment de la festiv ${ }^{t}$ de $S^{\text {ta }}$ Thecla corresponent als / Mussichs forasters. Lo qual $n^{\circ} \underline{79}$ Quo lecto / Dom $\operatorname{dix}^{t}$ Que se paguen las $7 £ 13$ ş de la $\mathrm{Adm}^{\circ}$ de / la Obra: Y sobre lo arreglo q $\mathrm{q}^{\mathrm{e}}$ se excita ab est motiu / dels salaris $\mathrm{q}^{\mathrm{e}}$ podrian señalarse als Mussichs / $\mathrm{q}^{\mathrm{e}}$ acostuman venir de defora $\mathrm{p}^{\mathrm{r}} \operatorname{dita}^{\mathrm{f}}$ festiv $\mathrm{v}^{\mathrm{t}} /$ Domi feren Com $^{\circ}$ als $\mathrm{S}^{\text {ors }}$ obrer y Penitt ${ }^{\mathrm{r}}$ a fi de $\mathrm{q}^{\mathrm{e}} /$ informantse ab lo $\mathrm{M}^{\mathrm{e}}$ de Capella exposian al / $\mathrm{m}^{\mathrm{t}} \mathrm{Yll}^{\mathrm{e}} \mathrm{Cap}^{\mathrm{l}}$ son dictamen."

[ 53 ] Pàg. 739 : «D. 27 7e 1777»: "D" Palomar propt Que havent entes de q e $^{\mathrm{e}}$ V.S havia / resolt $\mathrm{q}^{\mathrm{e}}$ lo obrer $\mathrm{S}^{\text {or }} \mathrm{Adm}^{\text {or }}$ de la Obra se encar- / regas de cobrar las porcions $\mathrm{q}^{\mathrm{e}}$ cedeix $\mathrm{D}^{\mathrm{n}}$ Ambros / Garcia $\mathrm{p}^{\mathrm{r}}$ Salari del Sub fa p[rese]nt a V.S de que / no se $\mathrm{p}^{\mathrm{r}}$ corresponent a son caracter lo encarre- / garsen un Cap ${ }^{\mathrm{r}}$ de estas mecanicas: sobre lo / q vostra V.S resoldrà lo q tinga $\mathrm{p}^{\mathrm{r}}$ con $^{\mathrm{t}}$. Et Dmi / Dix $\mathrm{q}^{\mathrm{e}}$ lo Apuntador o Boser deura recullir / los mencionats efectes q e cedeix Garcia, entregantlos / al S ${ }^{\text {or }}$ Obrer qian li aparega $\mathrm{q}^{\mathrm{e}}$ te alguna / quant proporcionador."

[ 54 ] Pàg. 742 : «D. 2 8 1777»: " $\mathrm{D}^{\mathrm{s}} \mathrm{Ll}^{\mathrm{s}}$ prop ${ }^{\mathrm{t}}$ Que havent reparat q $\mathrm{q}^{\mathrm{e}} \mathrm{lo} \mathrm{Com}^{1} /$ Sever Vila va vestit ab habits de Chor desde / sa casa a la Catth ${ }^{1}$ li ha aparegut ferho p[rese]nt / a V.S per si gusta pendrehi alguna provid / Et Domi Dix ${ }^{\mathrm{t}} \mathrm{q}^{\mathrm{e}} \mathrm{p}^{\mathrm{r}}$ ra no se innovia cosa alg.".

[ 55 ] Pàg. 745 : «D $108^{\mathrm{e}}$ 1777»: " $\mathrm{D}^{\mathrm{s}} \mathrm{Can}^{\mathrm{s}}$ Plana prop ${ }^{\mathrm{t}}$ Que havent mirat los exemplars / pera donar gracias a Deu $n^{\mathrm{e}} \mathrm{S}^{\text {or }}, \mathrm{p}^{\mathrm{r}}$ lo felis part / de la Princesa de Asturias, en conseq ${ }^{\mathrm{a}}$ de lo que / V.S resolgue, se ha trobat $q^{\mathrm{e}}$ se acostuma / cantar un solemne Te Deum y avisar al / Ajuntam ${ }^{\mathrm{t}}$ significantli $\mathrm{q}^{\mathrm{e}}$ lo diumenge proxim / segt se fara dita funcio despres de completas."

[ 56 ] Pàg. 749 : «D $188^{\mathrm{e}}$ 1777» : " $\mathrm{D}^{\mathrm{s}} \mathrm{Can}^{\mathrm{s}}$ Plana propt Que en att ${ }^{\mathrm{t}}$ a faltar en / esta Ygla lo primer

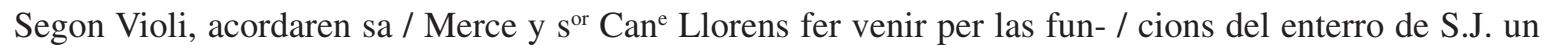
Violinista de Reus / asseñalantli ames de la porcio li tucas com a altre / dels Mussichs de ditas funcions dos reals de vuit / per lloguer de Cavalcadura $\gamma$ : ab lo animo de $\mathrm{q}^{\mathrm{e}}$ sas Merces pagarian estos en suposicio de / $q^{\mathrm{e}}$ V.S no ho tingues a be. Lo q ${ }^{\mathrm{e}}$ fa $\mathrm{p}$ [rese]nt a V.S / peraq elibere lo $\mathrm{q}^{\mathrm{e}}$ be li aparega. Et Dni deus ${ }^{\mathrm{t}}$ aprobarunt lo $\mathrm{q}^{\mathrm{e}}$ havian practicat dits / $\mathrm{S}^{\text {ors }} \mathrm{y} \operatorname{det}^{\mathrm{t}} \mathrm{q}^{\mathrm{e}}$ los dos reals de vuit se paguen / del [?] de gastos Com ${ }^{\mathrm{s}}$."

[ 57 ] Pàg. 756: «D $149^{\mathrm{e}}$ 1777»: " $\mathrm{D}^{\mathrm{s}} \mathrm{Can}^{\mathrm{s}}$ Plana prop ${ }^{t}$ Que hi ha un mem del / $\mathrm{R}^{\mathrm{t}} \mathrm{Jph}$ garcia altre del Susttentors de esta ygla / en lo qual ab lo motiu pretext de $q^{\mathrm{e}}$ se li proporciona / segons diu, alguna colllocacio de major convenencia / de la q $\mathrm{q}^{\mathrm{e}}$ gosa en esta Ygla, y de no tenir per / suficient la renda, $\mathrm{q}^{\mathrm{e}}$ actualm ${ }^{\mathrm{t}}$

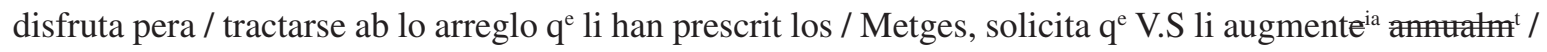
Cuyo mem ${ }^{1} \gamma \mathrm{n}^{\circ} 103$ Quo Lecto Domi dix ${ }^{\mathrm{t}}$ lectum. [...] [Pàg. 757 :] $\mathrm{D}^{\mathrm{s}}$ Can $^{\mathrm{s}}$ Plana prop ${ }^{\mathrm{t}}$ Que com a altre dels comisio / nats ab lo ${ }^{\text {or }}$ Obrer pera tractar lo concernent / als Mussichs de la Capella de esta Ygla fa $\mathrm{p}$ [rese]nt / a V.S haver trobat tenir $\mathrm{p}^{\mathrm{r}}$ molt convenient / lo substituhir a las plasas de primer segon / violi al fill del segon violi de Lleyda, asse- / nalantli ames de las $106 £ \mathrm{q}^{\mathrm{e}} \mathrm{ultim}^{\mathrm{t}}$ se / donavan a Anton Montana unas $40 £$ mes / annuals, y a Hernendes de Reus substituhirlo / a la plasa de Semmaner qe tenia Gasol ab / la obligacio de tocar lo violi S[em]pre $\mathrm{q}^{\mathrm{e}}$ se ofe- / resca y sia convenient asseñalantli $\mathrm{p}^{\mathrm{r}} \mathrm{sa} /$ manutencio annual ames de lo $\mathrm{q}^{\mathrm{e}}$ tenia dit / Gassol $\mathrm{p}^{\mathrm{r}}$ Semmaner aquella quan $\mathrm{q}^{\mathrm{e}}$ aparega / necessita annualm ${ }^{\mathrm{t}}$ pera prevenir que no molestia / despues a V.S pretenent augment de renda: Cuyo / proyecte podrá V.S resoldrer si se posara en / execucio; per lo q $\mathrm{e}^{\mathrm{e}}$ mira a un y altre dels / [Pàg. 758 :] dos proposats. Et Dom dix ${ }^{\mathrm{t}}$ que 
examinant previam ${ }^{\mathrm{t}}$ los referits $\mathrm{s}^{\text {ors }} \mathrm{Can}^{\mathrm{s}} \mathrm{Com}^{\mathrm{ts}}$ lo estat / en $\mathrm{q}^{\mathrm{e}}$ se troban las rendas de la $\mathrm{Adm}^{\mathrm{o}} \mathrm{de}$ la / Obra, posian en practica son proyecte si estas / poden suportarho."

[ 58 ] Pàg. 764 : «D $1 \mathrm{X}^{\mathrm{e}}$ 1777»: "D $\mathrm{D}^{\mathrm{s}} \mathrm{Can}^{\mathrm{s}}$ Plana prop ${ }^{\mathrm{t}}$ Que hi ha un mem¹/ del Com Rafiangel y Com $^{1}$ Marí $\mathrm{q}^{\mathrm{e}}$ com a Comissio ${ }^{\text {ts }} /$ de son Gremi representan queixarse alguns de sos / Individuos de $\mathrm{q}^{\mathrm{e}}$ $\mathrm{d}^{\mathrm{n}}$ Ambrosio Garcia Com ${ }^{1}$ y Sustt ${ }^{\text {or }} /$ de esta Ygla, no exercint lo ofici de Sustt ${ }^{\text {or }}$ ex- / cusia las funcions peculiars dels Comensals y algunas / en son cas fins dels mateixos ${ }^{\text {ors }} \mathrm{Cap}^{\mathrm{ls}}$ y Dignitaris / Sobre cuyo assumpto Sup ${ }^{\text {ca }} \mathrm{q}^{\mathrm{e}}$ V.S se servesca / providenciar lo $\mathrm{q}^{\mathrm{e}}$ tinga $\mathrm{p}^{\mathrm{r}}$ convenient [ Marge : "cuyo $\mathrm{m}^{1} \mathrm{n}^{\mathrm{o}} 110$ / Quo lecto "] Et Dni / tenint pnt dett ${ }^{\mathrm{e}} \mathrm{q}^{\mathrm{e}}$ interim dura la sede vac ${ }^{\mathrm{t}} /$ no se innove cosa alguna en esta depend ${ }^{\mathrm{a}}$ la que podra proposar lo Gremi de Comensals al futur s ${ }^{\text {or }} / \mathrm{Arch}^{\mathrm{e}}$ sise eompren pera determinarla."

\section{Any 1778}

[59] Pàg. 801 : «Dia 5 febrer 1778» : "D Plana propt : Que lo musich Pi ha tingut carta de son Pare en la que li comunica oferirli lo cap ${ }^{1}$ De Lley - / da $200 £$ annuals en virtud de la nova dosposicio / que han pres per la capella; y com comodia als dos / lo estan junts, si es dable [sic], ha demanat a sa mercé / q ammes de deliberar sobre esta insinuacio de son Pare, / estimaria fes pnt a V.S q e si tenia arbitre lo donar / plasa de viola, o violi a dit son pare ab lo mateix sa- / lari de $200 £ q^{\mathrm{e}}$ goza en Lleyda, rentun cediria la $\mathrm{q}^{\mathrm{e}} /$ te en aquella Igla per transferirse en esta, sobre tot / lo q fa pnt a VS a fi de $q^{\mathrm{e}}$ determinia lo $\mathrm{q}^{\mathrm{e}}$ li apa- / rega convenient. Et Dni, tenint pnts algunas cir- / cunstan ${ }^{\mathrm{s}}$ dels musichs $\mathrm{q}^{\mathrm{e}}$ actualm $\mathrm{m}^{\mathrm{t}}$ son a la capella, a / $\mathrm{q}^{\mathrm{e}}$ no se ha pogut providenciar ancara par lo atras / de compres del Quadern de la Obra, feren cominsió als $\mathrm{S}^{\text {rs }}$ / Cans, obrer, Penitencier y doctoral a fi de formar / dictamen pera terminar tots los rams de esta depen- / dencia, comunicant a S. Sria la resulta."

[ 60 ] Pàg. 804 : «Dia 17 Feb. 1778» : "D ${ }^{s} C^{s}{ }^{s}$ Ricci propt Que no obstant de ser / una de las obligs dels sustt ${ }^{\text {ors }}$ lo substituir o suplir / $\mathrm{p}^{\mathrm{r}}$ lo Semmaner $\mathrm{q}^{\mathrm{e}}$ faltia, comprenent sa Merce / $\mathrm{q}^{\mathrm{e}}$ alguns de ells tenen bastanta repugnancia / ho fa pnt perq ${ }^{\mathrm{e}}$ V.S se servesca resoldrer, si / ho fara observar $\mathrm{p}^{\mathrm{r}}$ aquell $\mathrm{Sustt}^{\text {or }}$ aqui toquia segons / sas ordina ${ }^{\mathrm{s}}$. Et Dni $\operatorname{dix}^{\mathrm{t}} \mathrm{q}^{\mathrm{e}}$ los $\mathrm{S}^{\text {ors }}$ Sind $^{\mathrm{s}}$ fa- / sian observar puntualm ${ }^{\mathrm{t}}$ lo Cap ${ }^{1}$ respectiu a esta / oblig ${ }^{\mathrm{o}}$ per lo Sustt ${ }^{\text {or }}$ aqui toquia suplir la falta / del Semmaner. Y havent lo $\mathrm{S}^{\text {or }} \mathrm{Can}^{\mathrm{e}}$ Penit $^{\mathrm{r}}$ / excitat la especie $\mathrm{q}^{\mathrm{e}}$ li comunica lo $\mathrm{S}^{\text {or }} \mathrm{M}^{\mathrm{e}} /$ de Capella desde Barna de trobar alli un / niño $\mathrm{q}^{\mathrm{e}}$ pot servir de Semmaner y tocar la / Viola spre $q^{\mathrm{e}}$ convinga, lo qual ha ofert transfe- / rirse a esta Ygla pera servir dits oficis, com / se li asseñalia un salari o renda ab la qual puga / mantenirse: trobantse Arnet en lo estat q no / ignora a V.S podra igualment resoldrer sobre esta / especie lo q li aparega convenient. Et Dni / dix ${ }^{\mathrm{t}}$ Que continuant a Arnet lo mateix salari / q eosa, se donaran al Semmaner sobredit / $30 £$ de salari cada any, ames de la renda / $\mathrm{q}^{\mathrm{e}}$ aixi per raho de semmaner pot guañar / residint, y servint a la Capella, component junta / dita renda 100 o $108 £$ cada any. Y ultim ${ }^{\mathrm{t}}$ havent dit $\mathrm{s}^{\mathrm{r}} \mathrm{Pen}^{\mathrm{r}}$ fer pnt $\mathrm{q}^{\mathrm{e}}$ seguidament se / podria resoldrer si se atendrá o no la / [Pàg. 805 :] solicitud del primer segon Violi de la Capella / Jpht Py y de son pare: Dom dix $^{\mathrm{t}} \mathrm{q}^{\mathrm{e}}$ per ara no te lloch una ni altra pretencio."

[ 61 ] Pàg. 810 : «Dia 27 feb. 1778»: " $D^{s}$ Can $^{s}$ Plana propt Que en seguida del qe V.S / havia de-

terminat sobre lo nou Semmaner que resideix en Barna, havia escrit al $\mathrm{M}^{\mathrm{e}}$ de / capella de esta $\mathrm{S}^{\mathrm{ta}} \mathrm{Ygla}, \mathrm{q}^{\mathrm{e}}$ 
se trobava en / aquella $\mathrm{Ciu}^{\mathrm{t}} \mathrm{y}$ havia diligenciat lo encontrar dit / Semmaner participantli q $\mathrm{e}$.S ames del salari / de Semmaner y del qe com a tal pot guañar / y ames del qe per tocar la viola podra gua- / nar en la Capella de Musica, li donarian $30 £$; peró que dit $\mathrm{M}^{\mathrm{e}}$ de capella li havia / respost, $\mathrm{q}^{\mathrm{e}}$ per est Salari no vindria, pero si en / cas se li augmentas unas $30 £$ mes; Lo q fa pnt / a V.S perq e se servesca deliberar lo $\mathrm{q}^{\mathrm{e}}$ tinga / $\mathrm{p}^{\mathrm{r}}$ convenient. Et Dni dix ${ }^{\mathrm{t}}$ Que se veigi si / se podra fer venir añadintseli unas $15 £$ o $20 £ /$ mes."

[ 62 ] Pàg. 816 : «D. 23 Mars 1778» : "D $\mathrm{DCan}^{\mathrm{s}} \mathrm{Llo}^{\mathrm{s}}$ prop $^{\mathrm{t}}$ Que se havia fet bastant notable / lo faltar lo $\mathrm{M}^{\mathrm{e}}$ de Capella lo dia de St Jph a las / completas, y no menos las vespres, per cuya falta / no se ha atrevit a multarlo, sens donarne part a V.S / [Pàg. 817 :] peraq $q^{\mathrm{e}}$ resolga lo $\mathrm{q}^{\mathrm{e}}$ tinga $\mathrm{p}^{\mathrm{r}}$ conv $^{\mathrm{t}}$. Et Dni atte- / nent a varias especies $\mathrm{q}^{\mathrm{e}}$ se excitaren concernents / a esta depend $\mathrm{d}^{\mathrm{a}}$ dett ${ }^{\mathrm{t}} \mathrm{pm} \theta=\mathrm{q}^{\mathrm{e}}$ los $\mathrm{s}^{\text {ors }}$ Sind $\mathrm{S}^{\mathrm{s}}$ se servescan / dir als $\mathrm{s}^{\text {or }} \mathrm{M}^{\mathrm{e}} \mathrm{q}^{\mathrm{e}}$ los entregui lo llibre de sas / obliga ${ }^{\mathrm{s}}$ de las de la Capella = seeundo y : Que en / quant a las faltas aixi del $\mathrm{M}^{\mathrm{e}}$ de Capella com / dels Susttentors prengan la provid ${ }^{\mathrm{a}} \mathrm{q}^{\mathrm{e}}$ los aparega / corresp $\mathrm{p}^{\mathrm{t}}$ segons la falta y sas circunstancias."

[ 63 ] Pàg. 818 : «D. 2 Apps 1778» : "D" Can Llo $^{s}$ prop ${ }^{\mathrm{t}}$ Que hi ha Carta / del R $\mathrm{R}^{\mathrm{t}}$ or Polio Benat de la Ygla Colegiata / de Siegni oferint remetrer a V.S set misas / impresas, tna de las y compostas en musica, una / de las quals es de difunts per lo import cada / una de ellas de 12 pesetas en plata, las quals / podran entregarse al $\mathrm{S}^{\text {or }}$ Bonandel en Barna / y en altres paratges $\mathrm{q}^{\mathrm{e}}$ señala a diferents / Subjectes; ab la Inteliga de $q^{\mathrm{e}}$ las remetra / ab dos vegadas diferents, entregantne 3 la / primera, y 4 la segona; y afi deno fer / tirar mes exemplars de las qe deuran re- / metrerse als subjectes, e Yglas q $q^{\mathrm{e}}$ hauran / firmat sa admisio, espera la resposta de V.S / per a saber sa determ ${ }^{\circ}$. Qual carta $\gamma 10$ / [Pàg. 819 :] Qua lee presa y notada en Ydioma Frances / conte substancialm ${ }^{t}$ lo sobredit, sobre lo que / podra V.S deliberar lo q e tinga $\mathrm{p}^{\mathrm{r}}$ convenient. / Et Dom dix $\mathrm{q}^{\mathrm{e}}$ se insertia ab los demes docu- / ments del corrent any Invenies in fine lig. $\operatorname{Sen}^{\circ} 10 / \mathrm{Y} \mathrm{q}^{\mathrm{e}}$ se responga al referit $\mathrm{R}^{\mathrm{t}}$ Polio que / estima lo $\mathrm{Cap}^{1} \mathrm{sa}_{\mathrm{att}}{ }^{\mathrm{y}} \mathrm{y} \mathrm{q}^{\mathrm{e}}$ no acomoda per ara sa $\mathrm{Sen}^{\mathrm{a}}$ lo subscriurerse admetent los exem- / plars sobredits."

[ 64 ] Pàg. 835 : «D. 30 Abl 1778»: " $\mathrm{D}^{\mathrm{s}} \mathrm{Can}^{\mathrm{s}} \mathrm{Llo}^{\mathrm{s}}$ prop $^{\mathrm{t}}$ Que havent reflexionat que/ al temps de fundarse la distrb ${ }^{\circ}$ de la Bosa can ${ }^{1}$ / se cantavan Matinas tots los dias a exeepeio uni - / eament dels ferials;

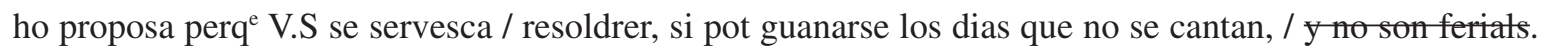
Et Dni suposant estar eompresa esta tenint present $\mathrm{q}^{\mathrm{e}}$ esta/ variacio en la visita fou feta $\mathrm{ab}$ aprob ${ }^{\circ}$ del $\mathrm{Y}^{\mathrm{m}}{ }^{\mathrm{m}}$ $\mathrm{S}^{\text {or }} \mathrm{D}^{\mathrm{n}}$ Joan Lario, $\mathrm{q}^{\mathrm{e}}$ de / gloria gose, y tenint pnt [ Margen : "qe sobre multa / cio de hora del / Matí a la tarde"] haver conegui del papa un / decret de aprobacio: Dix ${ }^{t}$ Que los S ${ }^{\text {ors }}$ Canonges / [Pàg. 836 :] de offici Comisionats pera examinar ab lo testam ${ }^{t} /$ del $S^{\text {or }}$ Robolledo los demes duptes proposats se / enterian de lo contengut en la aproba ${ }^{o}$ sobredita / y sens alterar interinam ${ }^{t}$ cosa alguna, se cantian / matines tots los dies de $1^{\mathrm{a}}$ y $2^{\mathrm{a}}$ clase, encarque / sian trasladats. $\mathrm{D}^{\mathrm{s}} \mathrm{Can}^{\mathrm{s}} \mathrm{Llo}^{\mathrm{s}}$ prop $^{\mathrm{t}}$ Que no menos dupta si pot V.S conti- / nuar a dir Matinas a la tarde en lo cas de no ser / aprobada esta variacio, sino $\mathrm{p}^{\mathrm{r}}$ lo $\mathrm{S}^{\text {or }} \mathrm{Arch}^{\mathrm{e}}$; lo qual se- / gons algunas doctrinas $q^{e}$ te pnt haver llegit solam / pot authorisar la expresada variacio de mati, a / la tarde ad temps, y durante causa, pero no / imperpertum; Per lo q si a V.S li apar be podran / igualm ${ }^{\mathrm{t}}$ examinar y ferse carrech de est dupte / los referits $\mathrm{S}^{\text {ors }} \mathrm{Can}^{\mathrm{s}}$ Comisionats, a fi de proposar- / lo a la Sag ${ }^{\mathrm{da}} \mathrm{Congreg}^{\mathrm{o}}$ o, al Papa, si ho judican / convenient, Y no se trobas compres en lo decret / de aprob ${ }^{\circ}$ sobrenotat : Et Dni dix ${ }^{t}$ placet. [ Marge : "Missa matutinal / Sobre no cantar- / se de algun temps / ha, estant a car- / rech dels 
Parroq" " ] D Can Ferrer prop ${ }^{\mathrm{t}}$ Que havent notat no / cantarse algun temps ha, la misa matinal, que / estava a car ${ }^{\mathrm{h}}$ del Parrals, e ignorar lo motiu de / esta tolerancia, ho fa pnt a fi de $\mathrm{q}^{\mathrm{e}}$ V.S prenga la provid $\mathrm{q}^{\mathrm{e}}$ li aparega convent. Et Dni dix ${ }^{\mathrm{t}}$ Que / los $\mathrm{S}^{\text {ors }}$ Sind $^{\mathrm{s}} \mathrm{y}$ demes Comisionats pera tractar ab / los Adv ${ }^{\mathrm{ts}}$ sobre la practica de las obligs dels Parls / comprenguian tambe, com a altre de ellas la / proposada."

[ 65 ] Pàg. 837 : «D. 4 Maig 1778»: " $\mathrm{D}^{\mathrm{s}} \mathrm{Can}^{\mathrm{s}} \mathrm{Llo}^{\mathrm{s}} \mathrm{prop}^{\mathrm{t}}$ Que havent notat faltar / dos dias lo Parl Amill a cantar las Lletanias / [Pàg. 838 :] sens prevenir altre q q $^{\mathrm{s}}$ splir sa obliga ${ }^{\mathrm{o}}$; ha enearrega / las feu posar en son puesto al Susttentor Jph Garcia / lo qual, ab lo modo de respondrerli en esta ocasio manifestá / bastanta repugnancia; Y enterantse ${ }^{\text {preguntant }}$ lomotiu $\mathrm{q}^{\mathrm{e}}$ havia / impedit al referit Par $^{1}$ Amill de acistir a la sobred $^{a}$ / obliga ${ }^{\circ}$ li digué $\mathrm{q}^{\mathrm{e}}$ la primera vegada fou per estar / ocupat en alguna cosa de son ministeri Parl, Y / la altra per estar de guarda, o vetlla ab un dels / dos Comensals qe estan extremunciats: Per lo q / no comprenent resistencia positiva en una ni / altra de las dos ocasions expresadas, no ha volgut / multarlo sens donarne part a V.S peraque / deliberia si se gratificara a Garcia, y si se / multara a Amill. Et Dni: Comprenent que / ningun motiu pot excusar al referit Par $^{1}$, pera / prevenir a altre $\mathrm{q}^{\mathrm{e}}$ substituesca sa oblig ${ }^{\circ}, \mathrm{o}$, donar / satisfacio correspon $^{\mathrm{t}}$ als s $\mathrm{s}^{\text {ors }}$ Sind $\mathrm{d}^{\mathrm{s}}$ luego de haverse / desocupat de la urgencia, $\mathrm{q}^{\mathrm{e}}$, lo impedis: $\operatorname{dett}^{\mathrm{t}} /$ Que pro hac vice no se multia; peró lo previnga / de las diligen omitidas, peraq $^{\mathrm{e}}$ tinga entes $\mathrm{q}^{\mathrm{e}}$ si en / altra ocasio, no las practica, se li aplicara la multa / correspon'; Y en quan a Garcia, $q^{\mathrm{e}}$ se considera / haverlo bastantam ${ }^{\mathrm{t}}$ grati- $^{\mathrm{t}}$ ficat $\mathrm{Sa} \mathrm{Sen}^{\mathrm{a}}$ desde / q $\mathrm{q}^{\mathrm{e}}$ comensa a servir a esta Ygla ab las repeti- / das quan ${ }^{\text {ts }} \mathrm{q}^{\mathrm{e}}$ graciosament li ha donat."

[ 66 ] Pàg. 851 : «Die 11 juny 1778» : "D Llorens prop ${ }^{t}$ Que hi ha un mem / del sustentor Jph garcia, acompañat de dos cer- / [Pàg. 852 :] tificacions de metges, que acreditan serli ne- / cessari lo mudar de ayres per millorar sa / salut, en virtut del que demana lo permis / per pasar â Oleria per tot lo temps que sia / menester, y que durant sa aus ${ }^{\mathrm{a}}$ V.S lo fase / reputar per present, qual sup[lenci] ${ }^{\mathrm{a}}$ si V.S gusta / se llegira. Dni legar, det est in fine $\mathrm{n}^{\circ} 35$ Qua lec- / ta Dni dixerunt, que despues de la octava de / Corpus se li dona permis per ausentarse dos / mesos, finits los quals, si continua sa causa, deu- / ra enviar certificat de dos Metges, y deliberara / lo $\mathrm{Cap}^{1}$ lo que li aparega convenient, pero que / desde lo primer dia que se ausentia, deura / deixar substitut que cumplia sas obligacions / pues altram ${ }^{t}$ lo posará sa Sria â expensas del / referit Garcia."

[ 67 ] Pàg. 867 : «D. 7 Aug A $^{\text {ti }} 1778 »:$ " $D^{s} C^{\text {Can }}$ Palomar prop" Que no essent ya de serva / lo major dels escolanets actuals, havia fet diliga en lo / $\mathrm{M}^{\mathrm{e}}$ de Capella pera buscar altre, y entre alguns $\mathrm{q}^{\mathrm{e}} / \mathrm{se}$ li han proporcionat, li apar, $\mathrm{q}^{\mathrm{e}}$ te $\mathrm{p}^{\mathrm{r}}$ ara millor / disposicio $\mathrm{q}^{\mathrm{e}}$ ningu de ells Thomas Granada, lo qual / espera, que V.S li donia lo permis pera entrar a / fer la prova acostumada. Et Dni Dix $\mathrm{q}^{\mathrm{e}}$ entre / Y havent entrat en compañia del $\mathrm{M}^{\mathrm{e}}$ de Capella / entoná per varios termes o punts. Quo audito: Dni / lo acceptaren $\mathrm{p}^{\mathrm{r}}$ altre dels escolanets de la Capella."

[ 68 ] Pàg. 880 : «D. 17 Se 1778»: " $\mathrm{D}^{\mathrm{s}} \mathrm{Can}^{\mathrm{s}} \mathrm{Llo}^{\mathrm{s}}$ propt Que esta format lo borro dels / edictes per la comensalia destinada $\mathrm{p}^{\mathrm{r}}$ lo organista / y si V.S gusta se llegiran junt ab la nota de algunas / refleccions, $q^{\text {e }}$ si apar be se añadiran pera evitar / controversia eb lo successiu: Dni Leg ${ }^{\mathrm{r}}$ [ Marge : "est n ${ }^{\mathrm{o}}$ 60"] Quibas / lectis: Dm dix ${ }^{t}$ : Que se procurian añadir totas / aquellas clausulas, $\mathrm{q}^{\mathrm{e}}$ poden obviar duptes y son / respectivas al organista actual, lo q e podra exe- / cutar lo Secretari Cap ${ }^{\mathrm{r}}$ comunicantho antes de / traurer los edictes a aquells $S^{\text {ors }}$ Canonges $\mathrm{q}^{\mathrm{e}}$ tinga / $\mathrm{p}^{\mathrm{r}}$ convenient a fi de veurer si falta alguna circunstancia." 


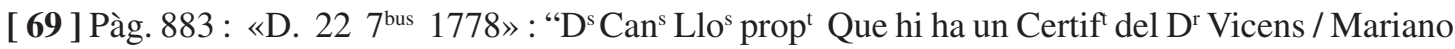
Hervás, lo qual afirma haverse administrat / lo Viattich al Susttentor Jph Garcia, ab lo qual apar / acreditar suficientment la justa causa de sa ausen ${ }^{\text {a }}$ [Pàg. 884 :] a fi de q e se li continue la pncia a horas Canonicas, y si / V.S gusta se intentara y se excutara de esta manera / Et Dni dix ${ }^{t}$ placet est $n^{\circ} \underline{63} . D^{s}$ Can $^{s}$ Llo $^{s}$ prop $^{t}$ Que tenint present de $\mathrm{q}^{\mathrm{e}}$ a $\mathrm{M}^{\mathrm{n}}$ Joan / ordeig se li dona las $\mathrm{Com}^{\mathrm{s}}$ Distribs per tenir lo ofici / bestinat a tocar lo Fogotot [sic] o Baixo, si a V.S / li apar be se li podra prevenir de q quant no hi haje / altre dels Mussichs q $\mathrm{q}^{\mathrm{e}}$ sapia tocarlo, que ho deguia practicar ell. Et Dni dix ${ }^{\mathrm{t}}$ placet. [...] $\mathrm{D}^{\mathrm{s}} \mathrm{Can}^{\mathrm{s}} \mathrm{Llo}^{\mathrm{s}}$ prop ${ }^{\mathrm{t}}$ Que si V.S gusta se llegira altra / vegada lo borro dels edictes del organista al q $q^{\mathrm{e}}$ se han añadit las / especies $\mathrm{q}^{\mathrm{e}}$ se excitaren en lo $\mathrm{Cap}^{1}$ antecedent, afi de / $\mathrm{q}^{\mathrm{e}}$ si apar be se puga donar a la Imprempta / y practicar las demes diligen $^{\mathrm{s}}$ previas a la provisio / de la Comensalia vac[an] destinada $\mathrm{p}^{\mathrm{r}}$ lo Organista. Dni / leg ${ }^{\mathrm{r}}$ Quo lecto: Dni dix ${ }^{t}$ placet, y que se remetia sola- / ment a las Yglas Cathedrals i Colegiatas de la Corona de Aragó."

[ 70 ] Pàg. 884 : «D. 2 8 1778»: " $\mathrm{D}^{\mathrm{s}} \mathrm{Can}^{\mathrm{s}} \mathrm{Llo}^{\mathrm{s}}$ prop $^{\mathrm{t}}$ Que hi ha la fe de obit del Rn / Jph Garcia Com $^{1}$ Sustt $^{r}$ de esta Igla q ${ }^{\mathrm{e}}$ si V.S gusta se / llegira: Dni leg ${ }^{\mathrm{r}}{ }^{\mathrm{o}} \underline{65}$ Qua lecta: Dni dix ${ }^{\mathrm{t}}$ Inseratur. [...] $\mathrm{D}^{\mathrm{s}} \mathrm{Can}^{\mathrm{s}}$ Ric prop ${ }^{t}$ Que si a V.S li apar se embiaran los / edictes $\mathrm{p}^{\mathrm{r}}$ la Comensalia vac ${ }^{\mathrm{t}} \mathrm{p}^{\mathrm{r}}$ mort de Gareia Jph Garcia / fentlos tirar los reimprimir com los del Organista en nom de V.S y dirigirlos / a las mateixas Yglas del Principat y Colegiatas de la Corona / de Arago. Et Dni dixt: Que se executia com se proposa / procurant $\mathrm{q}^{\mathrm{e}}$ quedian 8 dias de intermedi de uns examens / a altres, y añadint al edicte de la Sustentoria, $\mathrm{q}^{\mathrm{e}}$ en lo cas de no poder servir $\mathrm{p}^{\mathrm{r}}$ algun just impedim ${ }^{\mathrm{t}}$ son ofici, deura a expensas / suas posar un Substitut $\mathrm{q}^{\mathrm{e}}$ sia a sattisfa ${ }^{\circ}$ del cap.".

[ 71 ] Pàg. 893 : «D. 23 8re 1778»: " $\mathrm{D}^{\mathrm{s}}$ Plana prop ${ }^{\mathrm{t}}$ Que hi ha un mem ${ }^{1} \mathrm{p}^{\mathrm{r}}$ V.S / del Infantillo Pere Manubens, en $\mathrm{q}^{\mathrm{e}}$ demana alguna limosna pera socorrer la necessitat, en $\mathrm{q}^{\mathrm{e}}$ se en con- / tran sos Pares y tres Germans q $q^{\mathrm{e}}$ tots estan mals / Qual $\gamma \underline{73}$ Dni dix ${ }^{\mathrm{t}}$ lectam. D ${ }^{\mathrm{s}}$ Palomar prop ${ }^{\mathrm{t}}$ Que dias ha te per V.S un / mem ${ }^{1}$ de Jph Ricart Albeitart de Vilafranca / en qe exposa varias cosas, [ Marge : "relativas a la / pretencio qe te de / $\mathrm{q}^{\mathrm{e}}$ se vestesca al / fill $\mathrm{q}^{\mathrm{e}}$ actualm ${ }^{\mathrm{t}}$ ser- / veix de Monacillo / an la capella de / Mussichs de V.S ] q $\mathrm{q}^{\mathrm{e}}$ i V.S gusta $\gamma n^{\circ} 74$ Quo / [Pàg. 894 :] lecto: Dni determinarunt enterats de qe dit Monaeillo, nies, ni ha / estat de servey $\mathrm{p}^{\mathrm{r}}$ to offei de Monaeillo; y en conseq ${ }^{\mathrm{a}}$ q $\mathrm{q}^{\mathrm{e}}$ no lies deguda la gratifieacio, $\mathrm{q}^{\mathrm{e}}$ se ha acostumat / donar als Infantillos, $\mathrm{q}^{\mathrm{e}}$ han deixat $\mathrm{fo}^{\text {concluit son }}$ servey fins ara per / haver perdut la veu, esto es de ferlos un vestit / enter. Det' Que a dit Infantillo sel vestesea [ Marge : " $\mathrm{p}^{\mathrm{r}}$ tots Sants"] / se vestira-com als demes qe quedan en la Capella li facia lo vestit enter com se."

[ 72 ] Pàg. 895 : «D. 4 No 1778»: " $\mathrm{D}^{\mathrm{s}} \mathrm{Can}^{\mathrm{s}}$ Plana propt Que se han de anomenar / subgectes pera examinar als Concurrents a la oposicio / de la Comensalia destinada $\mathrm{p}^{\mathrm{r}}$ organista, aixi com / se hauran de anomenar tambe per la Sustentoria / vacant. Et Dom feren $\mathrm{Com}^{\mathrm{s}}$ als $\mathrm{S}^{\text {ors }}$ Sind $^{\mathrm{s}}$ Penit ${ }^{\mathrm{r}}$ / y Vilallonga pera fer venir un tercer examinador / [Pàg. 896 :] p pr lo Organista ames del $\mathbf{M}^{\mathrm{e}}$ de capella y de Joaquim / Vizente, donantlos plena facultat pera examinar y / arreglar las ordina ${ }^{\mathrm{s}} \mathrm{y}$ obliga $\mathrm{q}^{\mathrm{e}}$ deura cumplir / lo provist, y pera disposar tot lo concernent a estos / examens y als de la Sustentoria. [...] [Pàg. 897 :] D Dan $^{\mathrm{s}}$ Palomar prop ${ }^{t}$ Que lo $\mathrm{M}^{\mathrm{e}}$ de Capella / esta esperant lo permis de V.S pera entrar a pro- / var la veu de Benet Baixench, afi de $\mathrm{q}^{\mathrm{e}}$ / V.S determinia si se admetra per altre dels / Escolanets de la Capella. Et Dni dix $\mathrm{q}^{\mathrm{e}}$ entre; / y havent entrat, y entonat more solito. Domni / acceptaren, dient $\mathrm{q}^{\mathrm{e}}$ se previnga al $\mathrm{M}^{\mathrm{e}} \mathrm{de} / \mathrm{Capella}, \mathrm{q}^{\mathrm{e}}$ en lo 
successiu procuria buscar Miñons / de millor disp ${ }^{\circ}$ y esperansas sens presentarlos al Cap $^{1} / \mathrm{q}^{\mathrm{e}}$ no tinga una plena satisfacio de la sua veu."

[ 73 ] Pàg. 897 : «D. $14 \mathrm{No}^{\mathrm{e}}$ 1778» : "D Cans Plana propt Que haventse acabat lo termi- / ni prefigit en los edictes de la Comensalia vacant / [Pàg. 898 :] destinada $p^{r}$ lo organista de esta Metropa Ygla, y quedar / previngut un tercer Examinador, ames del $\mathrm{M}^{\mathrm{e}}$ de Capella, / y de Joaquim Viz ${ }^{\text {te }}$, si a V.S li apar be, se comensaran / los examens dema a la tarde despues de Completas. Et / Dni dix ${ }^{t}$ placet; y se inserten los edictes q $q^{\mathrm{e}}$ sunt in pre $\mathrm{n}^{\mathrm{0}} \underline{\mathbf{7 5}}$."

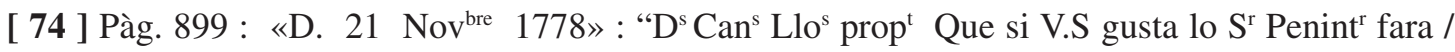
rela ${ }^{\circ}$ dels estat en que se eneontra la depend ${ }^{a}$ examens / y demes, concernent a la provisio de la Com ${ }^{a}$ vac $^{t}$ des- / tinada $\mathrm{p}^{\mathrm{r}}$ lo Organista a fi de $\mathrm{q}^{\mathrm{e}}$ despues se puga / elegir al opositor $\mathrm{q}^{\mathrm{e}}$ se comprenga mes benemerit. / En vista del que se ausenta de la Junta (adepta / prius pace) lo $\mathrm{S}^{\mathrm{r}} \mathrm{Can}^{\mathrm{e}}$ Foguet, renunciant son vot pera / acudir a algunas ocurrencias q $q^{\mathrm{e}}$ lo precisavan. Et Dni / Dix ${ }^{\mathrm{t}}$ placet: Y havent ohit la rela ${ }^{\mathrm{o}} \mathrm{del}^{\mathrm{r}} \mathrm{Penint}^{\mathrm{r}} / \mathrm{y}$ los dictamens separats dels tres examinadors / quals sunt in fine de $\mathrm{n}^{\circ} \underline{77}$ Dni votaren per / escutrini la referida Comen ${ }^{\mathrm{a}}$; Y havent tingut sis / vots Fran ${ }^{\mathrm{co}}$ Rodrigues, sinch Benet Junca, tres lo R $\mathrm{R}^{\mathrm{t}} \mathrm{Miq}[\mathrm{ue}]^{\mathrm{l}}$ Castello, y un lo $\mathrm{R}^{\mathrm{t}} \mathrm{Miq}[\mathrm{ue}]^{1}$ Basols, dels / quinse vocals q quedaren en la Junta $\mathrm{Cap}^{\mathrm{r}}$ / no verificantse tenir ningun opositor la major / part dels vots, resp $^{\circ}$ als vocals a son favor, se torna a fer segon escutrini, excloent a Basols / y a Miq[ue $]^{1}$ Lopez de la concurrencia passiva a / la eleccio, y quedá elegit Fran ${ }^{\text {co }}$ Rodriguez / als 8 vots, havent tingut Benet Junca 4 y / Miq[ue $]^{1}$ Castello 3 dels set restants: Pero antes / de comensar los escutrinis se confirma unanimam ${ }^{t}$ / viva voce la habilitacio y aprobacio de tots los / opositors referits en atten $^{\circ}$ a la distingida graduacio, que / tots meresqueren respectivam ${ }^{t}$ per los tres examina- / dors, donant facultat al S[ecreta]ri Cap ${ }^{r}$ perq $^{\mathrm{e}}$ donia / testimoni a cada hu de ells a la referida / [Pàg. 900 :] habilitacio, y graduacio sempre $\mathrm{q}^{\mathrm{e}}$ sia menester."

[ 75 ] Pàg. 901 : «D. 23 Nove 1778»: "D Cans Plana prop" Que los gastos ocorreguts pera / gratificar lo organista de la Selva altre dels examinadors / aixi com lo $\mathrm{q}^{\mathrm{e}}$ se pensa donar als de esta ygla, despues / de haverse finit los examens de la Sustentoria $\mathrm{vac}^{\mathrm{t}} \mathrm{ab} /$ lo permis de V.S, desitja saber si se pagara de la $\mathrm{Adm}^{\circ}$ de la Obra, o de q $\mathrm{q}^{\mathrm{e}}$ fondo. Et Dni dix que / se pague de la $\mathrm{Adm}^{\mathrm{o}}$ de la obra, y q $\mathrm{q}^{\mathrm{e}}$ los $\mathrm{S}^{\text {ors }} \mathrm{Can}^{\mathrm{s}} /$ Comisionats gratifiquen proporcionalm ${ }^{t}$ al treball als examinadors de esta Ygla."

[ 76 ] Pàg. 902 : «D. 27 9e 1778»: " D $^{\mathrm{s}} \mathrm{Can}^{\mathrm{s}} \mathrm{Llo}^{\mathrm{s}}$ Sind $^{\mathrm{s}}$ prop ${ }^{\mathrm{t}}$ Que haventse finit los $30 /$ dias qe se prefigiren de temps $\mathrm{p}^{\mathrm{r}}$ la oposicio a la / Soet Sostentoria vact, si a V.S li apar be se / [pàg. 903 :] comensaran los examens dema a la tarde despues de / Completas, y se finalisaran lo dilluns dia 31 del corrent / mes. Et Dni dix ${ }^{t}$ placet. $\mathrm{Id}^{\mathrm{m}}$ prop $^{\mathrm{t}}$ Que si ocorre lo compareixer algun altre / opositor despues de comensar los examens podra V.S re- / soldrer si se admetra. Et Dni dix $\mathrm{q}^{\mathrm{e}}$ se admetra. $\mathrm{Id}^{\mathrm{m}}$ prop ${ }^{\mathrm{t}}$ Que si algu dels opositors volgués solicitar alguna / subvencio o caritat per sa vinguda, o regres podrá V.S / resoldrer si admetra o no la suplica, o mem¹. Et Domi / dix ${ }^{\mathrm{e}}$ e se admetra."

[ 77 ] Pàg. 903 : «D. 3 X 1778» : "D Can Llo $^{\mathrm{e}}$ 17nd prop $^{\mathrm{t}}$ Que los examinadors han / entregat son dictamen sobre los opositors $\mathrm{q}^{\mathrm{e}}$ han concorregut / a la Susttentoria vact $\mathrm{p}^{\mathrm{r}}$ mort de Jph Garcia, y si V.S. / gusta se llegiran : Dmi legt in fine $\mathrm{n}^{\circ} 81$ Quibus / lectis: Dmi habilitaren uniformem ${ }^{t}$ a tots: $\mathrm{Y}$ haventse vo- / tat per escrutini, queda elegit Magi Danis y Figueres / $\mathrm{n}^{1}$ de la Ciu ${ }^{t}$ de Cervera, havent tingut deu vots 
dels 18 / vocals, q e concorregueren en la Junta Cap ${ }^{\mathrm{r}} 7$ Joan terrés / y 1 Ysidro Vagueria. ${ }^{\mathrm{s}} \mathrm{Can}^{\mathrm{s}}$ Llo $^{\mathrm{s}}$ Sind $^{\mathrm{s}}$ prop $^{t}$ Que en la suposició de $\mathrm{q}^{\mathrm{e}}$ hage pres / pos[ses]io lo referit Magi Danis de la Comensalia antes del / dia 8 dels corrts desitja saber, si se li pagara entesa / la Mesada com als demes Comensals o be solam ${ }^{\mathrm{t}}$ lo / $\mathrm{q}^{\mathrm{e}}$ correspon al pnt Mes de Ses ${ }^{\mathrm{e}}$ Et Dni dix ${ }^{\mathrm{t}}$ Que / se li pague lo q eorrespon al Desem solm $^{\mathrm{t}}$. D ${ }^{\mathrm{s}} \mathrm{Can}^{\mathrm{s}} \mathrm{Llo}^{\mathrm{s}}$ Sind $^{\mathrm{s}}$ prop $^{t}$ Que hi ha un mem ${ }^{1}$ de Alberto Viz $^{\text {te } / \text { altre dels opositors lo qual demana alguna subven }}{ }^{\circ}$ pera / regresar a son destino, y si V.S. gusta se llegira: Dni / $\operatorname{leg}^{\mathrm{r}} \mathrm{n}^{\mathrm{o}} 82$ Quo lecto: Dni dix ${ }^{\mathrm{t}}$ Que se li donen dos / [Pàg. 904 :] dobles del thecasi de gastos $\mathrm{Com}^{\mathrm{s}}$ y aixi mateix q queda / a la dispo dels $\mathrm{S}^{\text {ors }}$ Sinds lo donar del mateix thecasi als / demes $q^{\mathrm{e}}$ demanen lo $\mathrm{q}^{\mathrm{e}}$ los aparega corresp $\mathrm{p}^{\mathrm{t}}$ segons / sas circunstans."

[ 78 ] Pàg. 904 : «D. 5 Des $1778 »:$ "D ${ }^{\mathrm{e}} \mathrm{Can}^{\mathrm{s}} \mathrm{Llo}^{\mathrm{s}}$ Sind $^{\mathrm{s}}$ prop $^{t}$ Que lo Susttor Magi Danis / y Figueres enterat de sas obligas jura cumplirlas / en pncia de un dels Sindichs de V.S. devant del Nott / Blai y tes ${ }^{\mathrm{s}}$ lo dia 4 dels Corr ${ }^{\mathrm{s}}$ y seguidam ${ }^{\mathrm{t}}$ li feu / Colacio de la Comensalia lo $\mathrm{S}^{\text {or }} \mathrm{Can}^{\mathrm{e}} \mathrm{D}^{\mathrm{r}}$ Pere Jaume / Botines per expectar antes a la Cabiscolia y si V.S. / gusta se li donara pos[ses]io solutis solvendis. Et Dni / Dix ${ }^{t}$ placet."

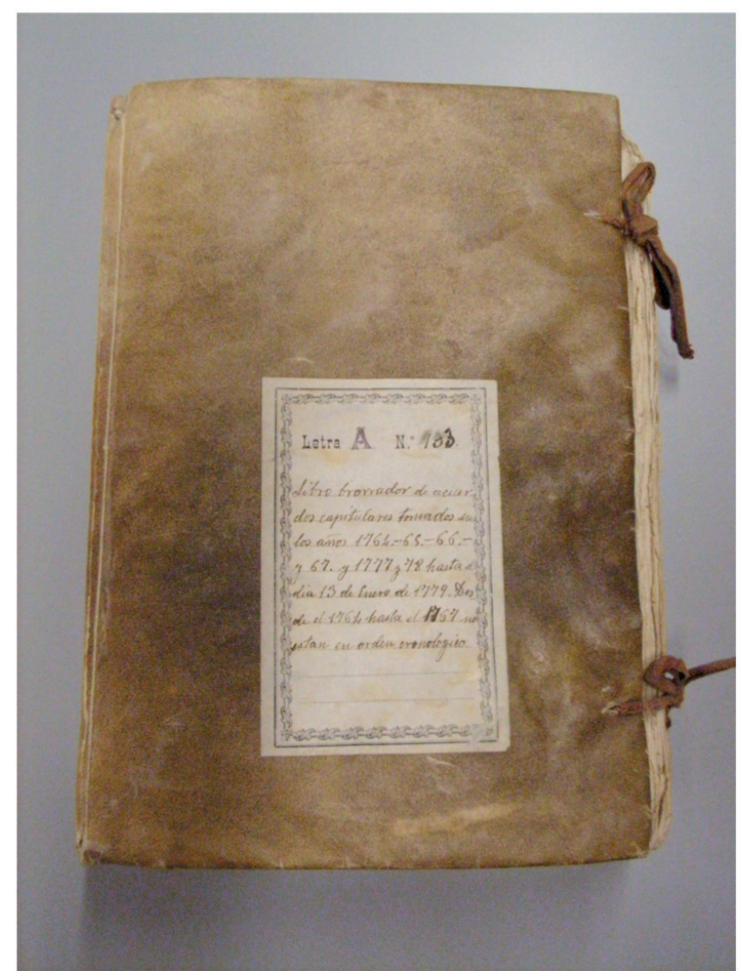

Figura 1. Llibre esborrany d'actes capitulars

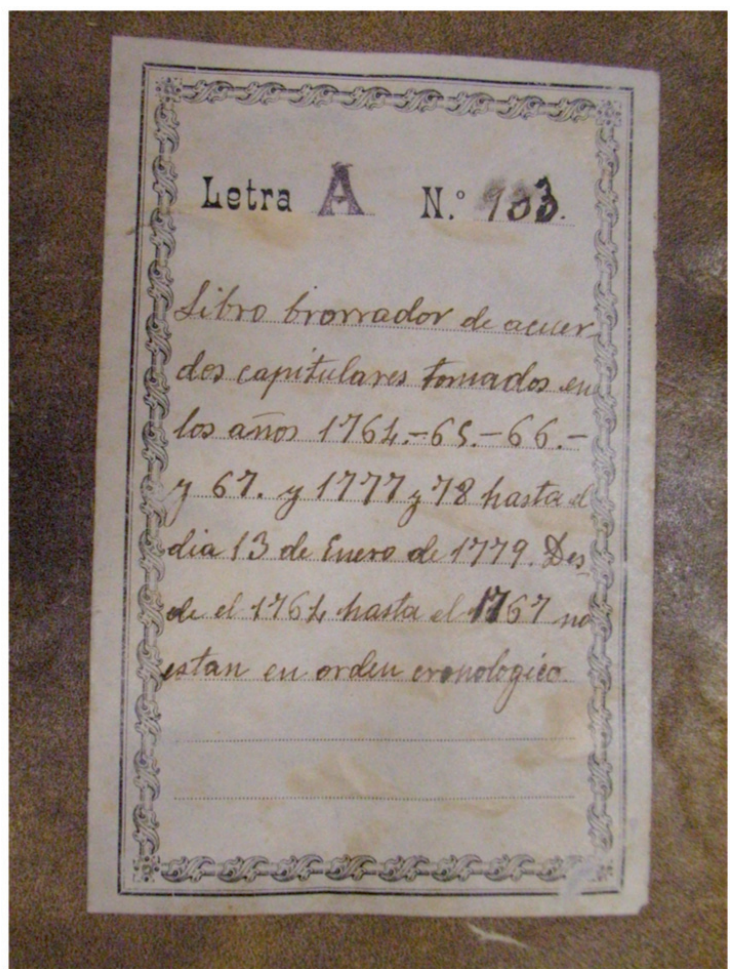

Figura 2. Etiqueta de la coberta 


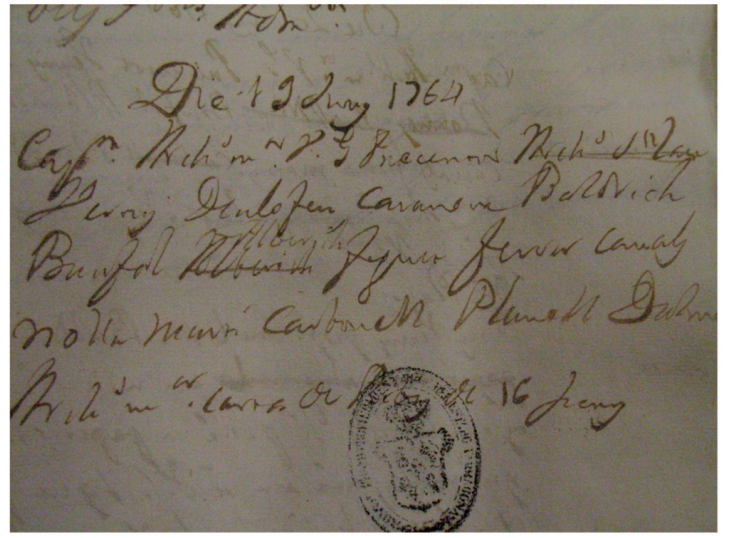

Figura 3. Exemple de grafia (19 de juny de 1764)

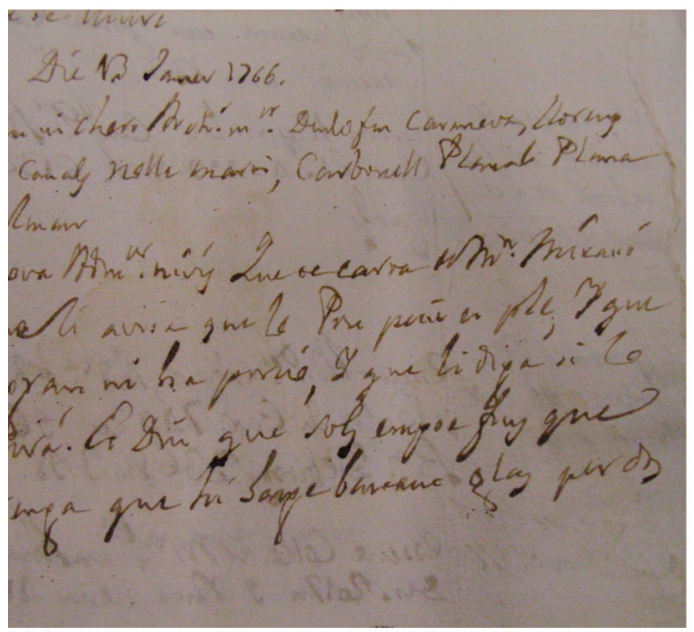

Figura 5. Exemple de grafia (13 de gener de 1766)

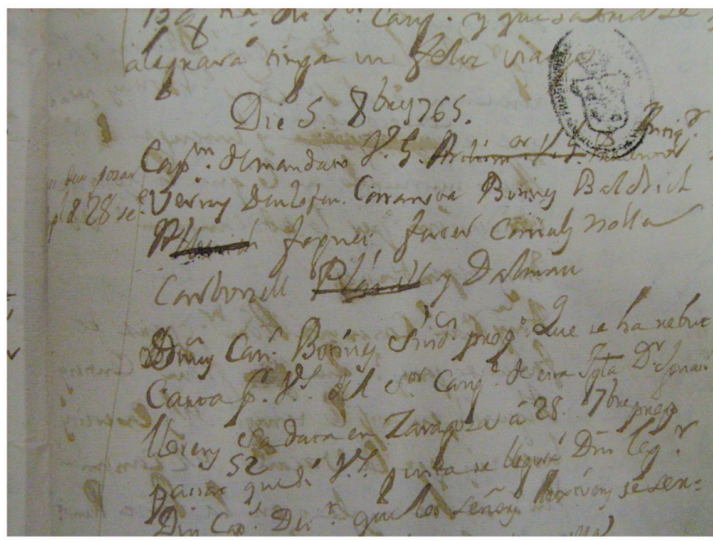

Figura 4. Exemple de grafia (5 d'octubre de 1765)

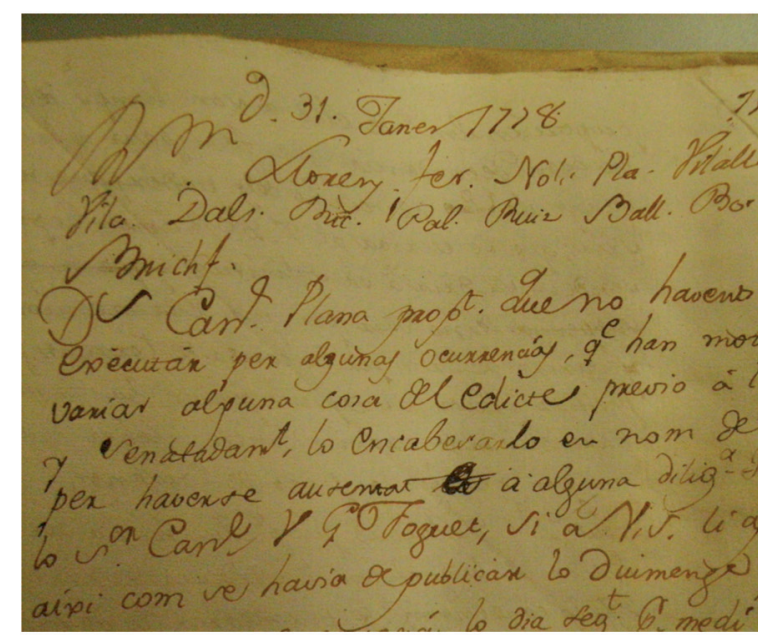

Figura 6. Exemple de grafia (31 de gener de 1778) 


\section{ÍNDEX ONOMÀSTIC}

ARMET, [?]; [vid. ARNET, [?]]; violó.

ARNET, [?]; violó; 4, 5, 15, 60

ARNIT, [?]; arpista; 22.

BARCELO, Fran ${ }^{\mathrm{co}}$; trompa i clarí; 18.

BASOLS $S^{8}$ Miquel; organista; 74.

CASTELLO, Miquel; organista; 74.

DANIS FIGUERES, Magí; sustentor; 77, 78.

ERNANDEZ, Fran ${ }^{\mathrm{co}}$; [vid. HERNANDEZ, Fran ${ }^{\mathrm{co}}$; músic de la capella].

FERRER, Jaume; sustentor; 16, 31.

FERRES, Joan; sustentor; 77.

GARCIA, Joseph; sustentor; 34, 44, 48, 49, 57, 65, 66, 69, 70, 77.

GARCIA DE LOS DOS, Ambrós; sustentor; 29, 30, 38, 39, 42, 43, 44, 46, 48, 50, 51, 53, 58.

GARCIA DE LOS DOS, Ambrosio; [vid. GARCIA DE LOS DOS, Ambrós; sustentor].

GASSOL, Anton; sustentor primer; 1, 7, 57.

GRANADA, Thomas; escolanet; 67.

GUASCH, Joseph; trompa i clarí; 18.

HERNANDEZ, Fran ${ }^{\mathrm{co}}$; músic de la capella; 6, 57.

HERNENDES, Fran ${ }^{\mathrm{co}}$; [vid. HERNANDEZ, Fran ${ }^{\mathrm{co}}$; músic de la capella].

JORDA, Pau; trompa primera; 41.

JUNCA, Benet; organista; 74.

LOPEZ, Miquel; organista; 74.

MALE, Joseph; monacillo; 8.

MANUBENS, Pere; infantillo; 70.

MIROSA, Ramon; sustentor; 34, 44, 48.

MONTAÑA, Anton; violí primer i segon; 1, 57.

N., Mathias; músic; 11

OLIVER, Joseph; escolanet; 2

ORDEIG, Joan; baixó; 15, 69.

ORDEIX, [?]; sustentor; 18.

PI, Joseph; violí primer i segon; 59, 60.

7 A continuació del cognom de cada músic, s'anota l'ofici pel qual és anomenat a les actes capitulars, o bé pel qual habitualment se l'identifica. Per "sustentor" s'ha d'entendre soxantre, el músic de la capella la funció principal del qual era entonar les salmòdies, antífones i salms i ensenyar als sustentors novicis. A més, tenien l'obligació de saber de cant pla i cant figurat i tocar l'instrument del qual tenien habilitat quan fes falta. No s'han regularitzat els noms i cognoms, que són citats tal com apareixen a la font. Quan un mateix músic apareix amb diferents grafies, s'indiquen les variants amb referències creuades. Els números a continuació de cada nom es refereixen al número d'entrada de cada acta capitular corresponent en què apareix el nom del músic.

8 En el cas d'opositors que es presentaven a la plaça sense èxit, els noms complets d'aquests s'han posat en cursiva. 
PY, Joseph; [vid. PI, Joseph; violí primer i segon].

RIPOLL, Andreu; cor; 45.

RODRIGUES, Fran ${ }^{\mathrm{co}}$; organista; 74.

SALVAT, Gaspar; sustentor; 44, 49.

SOLANELLAS, Joseph; escolanet; 2.

TERRES, Joan; sustentor; 77.

VAGUERIA, Isidro; sustentor; 50, 77.

VILA, Sever; sustentor; 44, 48, 49, 54.

VILA, Silverio; sustentor; 16.

VIZENTE, Alberto; sustentor; 77.

\section{ÍNDEX DE MATÈRIES}

ARPISTA; 22.

BAIXÓ; 69.

BAIXONISTA; 22.

CANT FIGURAT; 7, 11, 25, 27.

CANT PLA; 7, 11, 25, 27.

CAPELLA; 1, 7, 19, 23, 24, 27, 29, 60, 61, 62, 67, 71.

CLARÍ; 18.

COR / Chor; 1, 8, 22, 28, 29, 30, 35, 36, 41, 45, 47, 48, 51, 54.

ESCOLANET/ Infantillo / Monacillo / Monecillo; 1, 2, 8, 10, 23, 67, 71, 72.

FAGOT / Fogot / Fogotot; 18, 69.

MESTRE DE CAPELLA; 1, 3, 7, 11, 15, 21, 25, 27, 42, 52, 60, 62, 67, 72, 73.

MÚSICS DE LA CAPELLA; 6, 48, 57.

ORGANISTA; 1, 24, 41, 68, 69, 70, 72, 73, 74, 75.

ORGUE /orga; 1.

SUSTENTOR/ Susttentor / Succentor; 1, 7, 9, 11, 12, 14, 16, 17, 19, 21, 27, 28, 29, 30, 31, 32, 33, 34, 38, $39,41,42,44,46,48,49,50,57,58,60,62,66,69,70,78$.

SUSTENTORIA / Susttentoria / Succentoria; 9, 12, 25, 29, 32, 44, 70, 72, 75, 76, 77.

TROMPA; 18, 41.

VIOLA; 59, 60, 61.

VIOLÍ; 57, 59.

VIOLÍ PRIMER I SEGON; 1, 56, 57, 60.

VIOLINISTA; 56.

VIOLÓ; 4, 5.

XANTRE/ Chantre; 20. 


\section{BIBLIOGRAFIA}

BONASTRE I BERTRAN, Francesc, "La capella musical de la seu de Tarragona a mitjan segle XVIII", Boletín Arqueológico de la Societat Arqueològica Tarraconense, IV/133-140 (Tarragona, 19761977): 259-270.

CAZURRA BASTÉ, Anna, El compositor i mestre de capella Joan Rosell i la seva aportació al preclassicisme musical hispànic. Tesi doctoral, Universitat Autònoma de Barcelona, 1994.

FA ECHEVARRIA, Mayra, Melcior Juncà (1757-1824): biografia i música litúrgica. Tesi doctoral, Universitat Autònoma de Barcelona, 1999.

GREGORI CIFRÉ, Josep M., "Joan Crisòstom Ripollès (†1746): Biografia i catalogació de la producció musical conservada", Boletín Arqueológico de la Societat Arqueològica Tarraconense, IV (Tarragona, 1976-1977): 251-258.

GREGORI CIFRÉ, Josep M., "Joan Crisòstom Ripollès (ca.1680-1746), Mestre de capella de la catedral de Tarragona", Recerca musicològica, II (Barcelona, 1982): 19-41.

GREGORI CIFRÉ, Josep M., "Joan Crisòstom Ripollès (1678-1746) a les oposicions del magisteri de capella de la seu de Tortosa", Butlletí Arqueològic de la Reial Societat Arqueològica Tarraconense, V/14 (Tarragona, 1992): 137-147.

RAMON I VINYES, Salvador, "Canonges, comensalies, beneficis, mestres de capella i organistes de la catedral de Tarragona”, Butlletí Arqueològic de la Reial Societat Arqueològica Tarraconense, V/2122 (Tarragona, 1999): 241-595.

RIFÉ I SANTALÓ, Jordi, Els villancets d'Enmanuel Gònima, 1712-1792: un model de transició musical del barroc al preclassicisme a la Catalunya del segle XVIII. Tesi doctoral, Universitat Autònoma de Barcelona, 1992.

RUSIÑOL I PAUTES, M. del Carme, "Mira Piadosa Thecla, de Melcior Juncà (1757-1809): contribució a l'estudi del villancet català de les darreries del segle XVIII", Recerca musicològica, VIII (Barcelona, 1988): 143-191.

RUSIÑOL I PAUTES, M. del Carme, Els villancets de Melcior Juncà: contribució al seu estudi a la música catalana del segle XVIII. Tesi doctoral, Universitat Autònoma de Barcelona, 1994.

Recibido: 23/12/2010

Aceptado: 02/05/2012 